\title{
GAUSS-MANIN SYSTEMS OF FAMILIES OF LAURENT POLYNOMIALS AND A-HYPERGEOMETRIC SYSTEMS
}

\author{
THOMAS REICHELT AND ULI WALTHER
}

\begin{abstract}
In this note we study families of Gauß-Manin systems arising from Laurent polynomials with parametric coefficients under projection to the parameter space. For suitable matrices of exponent vectors, we exhibit a natural four-term exact sequence for which we then give an interpretation via generalized $A$-hypergeometric systems. We determine the extension groups from the parameter sheaf to the middle term of this sequence and show that the four-term sequence does not split. Auxiliary results include the computation of Ext and Tor groups of $A$-hypergeometric systems against the parameter sheaf.
\end{abstract}

\section{Contents}

Acknowledgememnts

1. Introduction

1.1. A-hypergeometric systems

1.2. $\mathcal{D}$-module functors

2. Restriction and de Rham functors of Euler-Koszul complexes

2.1. Local cohomology

2.2. Strongly resonant parameters

2.3. Restriction to the origin 8

2.4. De Rham cohomology 10

2.5. Ext and the polynomial solution functor 12

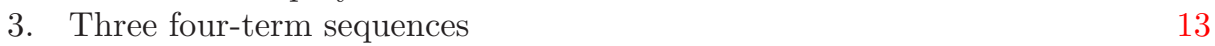

3.1. Quasi-equivariant bundles 14

3.2. The four-term sequence in terms of direct images 16

3.3. The four-term sequence with Gauß-Manin systems 17

3.4. Vanishing Gauß-Manin system and the extension class 22

References

\section{ACKNOWLEDGEMEMNTS}

This article grew out of a discussion with Duco van Straten who raised the question of the possible splitting of the sequence (3.4.1), see Theorem 3.13. We

1991 Mathematics Subject Classification. 13N10,14M25,32C38,32S40,33C70.

Key words and phrases. Gauss-Manin, toric, hypergeometric, Euler-Koszul, D-module, Laurent polynomial, equivariant, extension.

TR was supported by a DFG Emmy-Noether-Fellowship (RE 3567/1-1), UW was supported by the NSF under grant 1401392-DMS. . 
would like to express our sincere thanks to him and to Christian Sevenheck for generously sharing their ideas.

\section{INTRODUCTION}

During the 1980s Gel'fand, Graev, Kapranov and Zelevinskiu introduced a class of systems of complex partial differential equations which are a vast generalization of the Gauß hypergeometric equation and which are nowadays known as $A$ hypergeometric (or GKZ) systems (cf. [GGZ87, GZK89] and a string of other articles of that period). Such an $A$-hypergeometric system has a hybrid combinatorial and algebraic flavor, its initial datum being an integer matrix $A$ and a parameter vector $\beta$ in the column space of $A$. This determines a left ideal $H_{A}(\beta)$ in the Weyl algebra $D$ and the $A$-hypergeometric system with respect to $A$ and $\beta$ is then the cyclic left $D$-module $M_{A}^{\beta}:=D / H_{A}(\beta)$. From this definition it is far from clear that these systems have any geometric interpretation.

The analytic behavior of $M_{A}^{\beta}$ (as a system of PDEs) is highly dependent on the parameter vector $\beta$. A technique to study this dependence, the Euler-Koszul functor, was developed by Matusevich, Miller and the second author in [MMW05]. This is a functor from the category of toric modules, which are a mild generalization of $\mathbb{Z} A$-graded $\mathbb{C}[\mathbb{N} A]$ modules, to the category of complexes of $D$-modules. The construction of this functor generalizes the Euler-Koszul complex on the semigroup ring $\mathbb{C}[\mathbb{N} A]$ (already known to Gel'fand, Kapranov and Zelevinskiı̌, [GZK89]) and was inspired by it.

The Euler Koszul complex provides a $D$-resolution of the corresponding $A$ hypergeometric system provided $\beta$ does not lie in the $A$-exceptional locus, defined via the local cohomology of $\mathbb{C}[\mathbb{N} A]$. An important step in the geometric interpretation of $A$-hypergeometric systems was achieved by Schulze and the second author in [SW09], generalizing work of Gel'fand et al. There they showed, using the EulerKoszul complex, that the Fourier-Laplace transform of $M_{A}^{\beta}$ can be identifed with the direct image of a twisted structure sheaf on a torus under a monomial map (depending on $A$ ) to affine space whenever $\beta$ is outside the set of strongly resonant parameters.

If $A$ is homogeneous, i.e. if $(1, \ldots, 1)$ is in its row span, then this embedding descends to an embedding of a torus of dimension one less into projective space. It was realized by Brylinski [Bry86] that the Fourier-Laplace transform of a $D$-module on affine space which is constant on all punctured lines through the origin can be expressed by a Radon transform of the corresponding $D$-module on the projective space.

Using this Radon transform the first author showed in [Rei14] that homogeneous $A$-hypergeometric systems with not strongly resonant, but integer parameter vector $\beta$ carry the structure of a mixed Hodge module. Furthermore, there exists a morphism to $M_{A}^{\beta}$ from the Gauß-Manin system of the maximal family of Laurent polynomials with Newton polytope equal to the convex hull of the columns of $A$. This map has $\mathcal{O}$-free kernel and cokernel, and is compatible with the natural mixed Hodge module structure on the Gauß-Manin system and on $M_{A}^{\beta}$ respectively.

Since $M_{A}^{\beta}$ is the terminal Euler-Koszul homology of the semigroup ring $\mathbb{C}[\mathbb{N} A]$ one wonders whether the Euler-Koszul homology of other toric modules (for example, $A$-graded ideals of $\mathbb{C}[\mathbb{N} A]$ ) carry a natural mixed Hodge module structure as well. In this paper we consider the maximal graded ideal of $\mathbb{C}[\mathbb{N} A]$ and prove 
that its terminal Euler-Koszul homology is isomorphic to the Gauß-Manin system of a map whose fibers are the complement of the fibers of the Laurent polynomial alluded to above.

We now give a short overview of the content of this article. In the first section we review the definition of $A$-hypergeometric systems, of the Euler-Koszul complex, and several functors on $D$-modules. In the following section we compute the restriction of $M_{A}^{\beta}$ to the origin, its de Rham cohomology, and the groups $\operatorname{Ext}_{D}^{\bullet}\left(\mathcal{M}_{A}^{\beta}, \mathcal{O}\right)$. A novel feature of this article is that we work throughout over any field of characteristic zero, rather than specifically over $\mathbb{C}$. In this more general setting we (re)prove that for not strongly resonant parameter the Fourier-Laplace transformed $A$-hypergeometric system can be viewed as the direct image of a twisted structure sheaf under a torus embedding. In the third section we show that the long exact Euler-Koszul homology sequences induced by the inclusion of the maximal graded ideal in $\mathbb{C}[\mathbb{N} A]$ is isomorphic to certain Gauß-Manin systems coming from a family of Laurent polynomials and compare this sequence with the sequence obtained in [Rei14].

1.1. A-hypergeometric systems. We introduce here the main notation and review some basis facts on $A$-hypergeometric systems and the Euler-Koszul functor. We refer to [MMW05, SW09] for more details.

Notation 1.1. Throughout, we work over the field $\mathbb{k}$ of characteristic zero.

In general we adopt the convention that we denote a sheaf by a calligraphic letter such as $\mathcal{M}$, a module by an Italic letter such as $M$, and categories and functors by Roman letters such as M.

Notation 1.2. Throughout, $A$ will be an integer matrix that we assume to be pointed: there should be a $\mathbb{Z}$-linear functional on the column space of $A$ that evaluates positively on each column of $A$.

For any integer matrix $A$, let $\mathbb{k}^{A}$ be a vector space with basis corresponding to the columns $\left\{\mathbf{a}_{j}\right\}_{j}$ of $A$. Let $R_{A}$ (resp. $O_{A}$ ) be the polynomial ring over $\mathbb{k}$ generated by the variables $\partial_{A}=\left\{\partial_{j}\right\}_{j}$ (resp. $x_{A}=\left\{x_{j}\right\}_{j}$ ) corresponding to $\left\{\mathbf{a}_{j}\right\}_{j}$; we read $R_{A}$ as coordinate ring on the variety $X_{A}:=\mathbb{k}^{A}$. Further, let $D_{A}$ be the ring of $\mathbb{k}$-linear differential operators on $O_{A}$, where we identify $\frac{\partial}{\partial x_{j}}$ with $\partial_{j}$ so that both $R_{A}$ and $O_{A}$ are subrings of $D_{A}$.

For any (semi)ring of coefficients $C$ we write $C A$ for the set of $C$-linear combinations of the columns of $A$. In particular, $\mathbb{k} A$ is a vector space.

Definition 1.3. Let $A$ be an integer matrix with independent rows whose $\mathbb{Z}$-ideal of maximal minors equals $\mathbb{Z}$.

For the parameter $\beta \in \mathbb{k} A$ let $H_{A}(\beta)$ be the $D_{A}$-ideal generated by the homogeneity equations

$$
\left\{E_{i} \bullet \phi=\beta_{i} \cdot \phi\right\}_{i}
$$

together with the toric partial differential equations

$$
\left\{\left(\partial_{A}^{\mathbf{v}_{+}}-\partial_{A}^{\mathbf{v}_{-}}\right) \bullet \phi=0 \quad \mid \quad A \cdot \mathbf{v}=0\right\},
$$

using (throughout) multi-index notation. Here, with $\mathbf{0}_{A}=(0, \ldots, 0)$ in $\mathbb{k}^{A}$, we write $E_{i}:=\sum_{j} a_{i j} x_{j} \partial_{j}$ and $\mathbf{v}_{+}=\max \left(\mathbf{v}, \mathbf{0}_{A}\right), \mathbf{v}_{-}=-\min \left(\mathbf{v}, \mathbf{0}_{A}\right)$. We put

$$
M_{A}^{\beta}:=D_{A} / H_{A}(\beta) \text {. }
$$


We have

$$
\begin{aligned}
x^{\mathbf{u}} E_{i}-E_{i} x^{\mathbf{u}} & =-(A \cdot \mathbf{u})_{i} x^{\mathbf{u}}, \\
\partial^{\mathbf{u}} E_{i}-E_{i} \partial^{\mathbf{u}} & =(A \cdot \mathbf{u})_{i} \partial^{\mathbf{u}} .
\end{aligned}
$$

The $A$-degree function (with values in $\mathbb{Z} A$ ) on $R_{A}$ and $D_{A}$ is:

$$
-\operatorname{deg}_{A}\left(x_{j}\right):=\mathbf{a}_{j}=: \operatorname{deg}_{A}\left(\partial_{j}\right) .
$$

We denote $\operatorname{deg}_{A, i}(-)$ the degree function associated to the weight given by the $i$-th row of $A$. Then $E_{i} P=P\left(E_{i}-\operatorname{deg}_{A, i}(P)\right) P$ for $A$-graded $P$. Let

$$
\varepsilon_{A}:=\sum_{j} \operatorname{deg}_{A}\left(\partial_{j}\right)=\sum_{j} \mathbf{a}_{j} .
$$

Let $M$ be an $A$-graded $D_{A}$-module. There are commuting $D_{A}$-linear endomorphisms $E_{i}$ via

$$
E_{i} \circ m:=\left(E_{i}+\operatorname{deg}_{i}(m)\right) \cdot m .
$$

for $A$-graded $m \in M$. In particular, if $N$ is an $A$-graded $R_{A}$-module one obtains

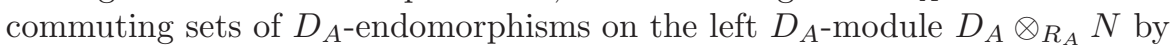

$$
E_{i} \circ(P \otimes Q):=\left(E_{i}+\operatorname{deg}_{i}(P)+\operatorname{deg}_{i}(Q)\right) P \otimes Q .
$$

The Euler-Koszul complex $K_{\bullet}(N ; E-\beta)$ of the $A$-graded module $N$ is the homological Koszul complex induced by $E-\beta:=\left\{\left(E_{i}-\beta_{i}\right) \circ\right\}_{i}$ on $D_{A} \otimes_{R_{A}} N$. In particular, the terminal module $D_{A} \otimes_{R_{A}} N$ sits in cohomological degree zero. We denote $\mathcal{K}_{\bullet}(N ; E-\beta)$ the corresponding complex of quasi-coherent sheaves. The cohomology objects are $H_{\bullet}(N ; E-\beta)$ and $\mathcal{H}_{\bullet}(N ; E-\beta)$ respectively. If $N(\alpha)$ denotes the usual shift-of-degree functor on the category of graded $R_{A}$-modules, then $K_{\bullet}(N ; E-\beta)(\alpha)$ and $K_{\bullet}(N(\alpha) ; E-\beta+\alpha)$ are identical.

Identifying $\mathbb{Z} A$ with $\mathbb{Z}^{\mathrm{rk}(A)}$ we get coordinates $\left\{t_{i}\right\}_{i}$ on $T_{A}=\operatorname{Spec}(\mathbb{k}[\mathbb{Z} A])=$ $\operatorname{Spec}\left(\mathbb{k}\left[\left\{t_{i}^{ \pm}\right\}_{i}\right]\right)$ and then an embedding

$$
h_{A}: T_{A} \longrightarrow \operatorname{Spec}\left(\mathbb{C}\left[\left\{\partial_{j}\right\}_{j}\right]\right)=\mathbb{k}^{A}
$$

induced by the monomial morphism

$$
t:=\left\{t_{i}\right\}_{i} \longrightarrow\left\{\prod_{i} t_{i}^{\mathbf{a}_{i j}}\right\}_{j}=: t^{A}
$$

The closure of the image of $h_{A}$ in $X_{A}$ becomes a toric variety via $h_{A}$ and is defined by the $R_{A}$-ideal $I_{A}$ given as the kernel of (1.1.2) and generated by all binomials $\partial_{A}^{\mathbf{v}_{+}}-\partial_{A}^{\mathbf{v}_{-}}$where $A \mathbf{v}=0$. We denote the semigroup ring

$$
S_{A}:=R_{A} / I_{A} \simeq \mathbb{k}[\mathbb{N} A] .
$$

We denote $\widetilde{\mathbb{N} A}$ the saturation of $\mathbb{N} A$ and by $\tilde{S}_{A}$ the associated semigroup ring, identical with the normalization of $S_{A}$.

The faces $\tau$ of the rational polyhedral cone $\mathbb{R}_{+} A$, i.e. the subsets of (the columns of) $A$ that minimize (over $A$ ) some linear functional $\mathbb{Z} A \longrightarrow \mathbb{Z}$, correspond to $A$ graded prime ideals $I_{A}^{\tau}$ of $R_{A}$ with $I_{A}^{\tau}=I_{A}+R_{A}\left(\left\{\partial_{j}\right\}_{j \notin \tau}\right)$. We let $R_{A} \partial_{A}$ be the unique $A$-graded maximal $R_{A}$-ideal.

An $R_{A}$-module $N$ is toric if it is $A$-graded, and if it has a (finite) $A$-graded composition chain

$$
0=N_{0} \subsetneq N_{1} \subseteq N_{2} \cdots \subsetneq N_{k}=N
$$


such that each composition factor $N_{i} / N_{i-1}$ is isomorphic as $A$-graded $R_{A}$-module to a face ring $R_{A} / I_{A}^{\tau}$ or one of its shifts by an element of $\mathbb{Z} A$.

For a finitely generated $A$-graded $R_{A}$-module $N=\bigoplus_{\alpha \in \mathbb{Z} A} N_{\alpha}$, let

$$
\begin{aligned}
\operatorname{deg}_{A}(N) & =\left\{\alpha \in \mathbb{Z} A \mid N_{\alpha} \neq 0\right\}, \\
\operatorname{qdeg}_{A}(N) & ={\overline{\operatorname{deg}_{A}(N)}}^{Z a r},
\end{aligned}
$$

the latter being the Zariski closure of the former in $\mathbb{k} A=\mathbb{k}_{\mathbb{Z}} \otimes_{\mathbb{Z}} \mathbb{Z} A$. For unions of such modules, degrees as well as quasi-degrees are defined to be the corresponding unions, compare [SW09].

Let $N=\mathbb{k}(-\alpha)$ be the graded $R_{A}$-module whose module structure is that of $R_{A} / I_{A}^{\emptyset}=R_{A} / R_{A} \partial_{A} \simeq \mathbb{k}$, and which lives entirely inside degree $\alpha \in \mathbb{Z}^{d}$. Then $K_{\bullet}(N ; E-\beta)$ is an exact complex if $\beta \neq \alpha$, and its differentials are zero otherwise.

Definition 1.4. If the row span of $A$ contains $\mathbf{1}_{A}$ we call $A$ homogeneous. Homogeneity is equivalent to $I_{A}$ defining a projective variety, and to the system $H_{A}(\beta)$ having only regular singularities [SW08].

1.2. $\mathcal{D}$-module functors. Let $X$ be a smooth algebraic $\mathbb{k}$-variety of dimension $d_{X}$. We denote by $\mathcal{D}_{X}$ the sheaf of algebraic differential operators and by $D_{X}$ its ring of global sections. For $X=\mathbb{A}^{n}$ we sometimes write $D_{n}$. We denote by $\operatorname{Mod}\left(\mathcal{D}_{X}\right)$ the Abelian category of left $\mathcal{D}_{X}$-modules. The full triangulated subcategories of the derived category $\mathrm{D}^{b}\left(\mathcal{D}_{X}\right):=\mathrm{D}^{b}\left(\operatorname{Mod}\left(\mathcal{D}_{X}\right)\right)$ consisting of objects with $\mathcal{O}_{X}$-quasicoherent (resp. holonomic) cohomology are denoted by $\mathrm{D}_{q c}^{b}\left(\mathcal{D}_{X}\right)\left(\right.$ resp. $\left.\mathrm{D}_{h}^{b}\left(\mathcal{D}_{X}\right)\right)$.

We recall the notation for cohomological shifting a complex $C^{\bullet}: C^{\bullet}[1]$ is the complex $C^{\bullet}$ shifted one step left, $\left(C^{\bullet}[1]\right)^{i}=C^{i+1}$, with corresponding shift of the morphisms.

Let $f: X \rightarrow Y$ be a map between smooth algebraic varieties. Let $\mathcal{M} \in \mathrm{D}_{q c}^{b}\left(\mathcal{D}_{X}\right)$ and $\mathcal{N} \in \mathrm{D}_{q c}^{b}\left(\mathcal{D}_{Y}\right)$, then we denote by

$$
f_{+} \mathcal{M}:=\mathrm{R} f_{*}\left(\mathcal{D}_{Y \leftarrow X} \stackrel{L}{\otimes} \mathcal{M}\right) \quad \text { and } \quad f^{+} \mathcal{N}:=\mathcal{D}_{X \rightarrow Y} \stackrel{L}{\otimes} f^{-1} \mathcal{N}\left[d_{X}-d_{Y}\right]
$$

the direct and inverse image functors for $\mathcal{D}$-modules; both preserve holonomicity and if $f$ is non-characteristic with respect to $\mathcal{N}$ then $f^{+}$is exact (up to a shift), (see e.g. [HTT08, Def. 2.4.2 \& Thm 2.4.6]). We denote by

$$
\begin{aligned}
\mathbb{D}: \mathrm{D}_{h}^{b}\left(\mathcal{D}_{X}\right) & \longrightarrow\left(\mathrm{D}_{h}^{b}\left(\mathcal{D}_{X}\right)\right)^{\text {opp }} \\
\mathcal{M} & \mapsto \mathrm{RHom}\left(\mathcal{M}, \mathcal{D}_{X} \otimes_{\mathcal{O}_{X}} \Omega_{X}^{\otimes-1}\right)\left[d_{X}\right]
\end{aligned}
$$

the duality functor, which also preserves holonomicity. We additionally define the functors

$$
f_{\dagger}:=\mathbb{D} \circ f_{+} \circ \mathbb{D} \quad \text { and } \quad f^{\dagger}:=\mathbb{D} \circ f^{+} \circ \mathbb{D} \text {. }
$$

If $X$ is an affine variety, we have an equivalence of categories

$$
\begin{aligned}
\operatorname{Mod}\left(\mathcal{D}_{X}\right) & \longrightarrow \operatorname{Mod}\left(D_{X}\right) \\
\mathcal{M} & \mapsto M:=\Gamma(X, \mathcal{M})
\end{aligned}
$$

where $\operatorname{Mod}\left(D_{X}\right)$ is the category of left $D_{X}$-modules.

Definition 1.5. Let

$$
\langle-,-\rangle: \mathbb{A}^{\ell} \times \hat{\mathbb{A}}^{\ell} \rightarrow \mathbb{A}^{1}, \quad\left(\lambda_{1}, \ldots, \lambda_{\ell}, \mu_{1}, \ldots \mu_{\ell}\right) \mapsto \sum_{i=1}^{\ell} \lambda_{i} \mu_{i} .
$$


(Here, and elsewhere, $\hat{\mathbb{A}}^{\ell}$ denotes an affine space of dimension $\ell$; we use the "hat" to keep apart source and range of the two functors defined in (1.2.2) below). Now define two $\mathcal{D}_{\mathbb{A}^{\ell} \times \hat{\mathbb{A}}^{\ell}}$-modules by

$$
\mathcal{L}:=\mathcal{O}_{\mathbb{A}^{\ell} \times \hat{\mathbb{A}}^{\ell}} e^{\langle\cdot, \cdot\rangle}, \quad \overline{\mathcal{L}}:=\mathcal{O}_{\mathbb{A}^{\ell} \times \hat{\mathbb{A}}^{\ell}} e^{-\langle\cdot, \cdot\rangle} .
$$

We refer to [KS97, Section 5] for details on these sheaves. Denote by $p_{1}: \mathbb{A}^{\ell} \times \hat{\mathbb{A}}^{\ell} \rightarrow$ $\mathbb{A}^{\ell}$ and $p_{2}: \mathbb{A}^{\ell} \times \hat{\mathbb{A}}^{\ell} \rightarrow \hat{\mathbb{A}}^{\ell}$ the projection to the first and second factors respectively. The Fourier-Laplace transform is defined by

$$
\begin{aligned}
\mathrm{FL}: \mathrm{D}_{q c}^{b}\left(\mathcal{D}_{\mathbb{A}^{\ell}}\right) & \longrightarrow \mathrm{D}_{q c}^{b}\left(\mathcal{D}_{\hat{\mathbb{A}}^{\ell}}\right) \\
M & \mapsto p_{2+}\left(p_{1}^{+} M \stackrel{L}{\otimes} \mathcal{L}\right)
\end{aligned}
$$

and

$$
\begin{aligned}
\mathrm{FL}^{-1}: \mathrm{D}_{q c}^{b}\left(\mathcal{D}_{\hat{\mathbb{A}}^{\ell}}\right) & \longrightarrow \mathrm{D}_{q c}^{b}\left(\mathcal{D}_{\mathbb{A}^{\ell}}\right) \\
M & \mapsto p_{1+}\left(p_{2}^{+} M \stackrel{L}{\otimes} \overline{\mathcal{L}}\right)
\end{aligned}
$$

Then $\mathrm{FL}^{-1} \circ \mathrm{FL}(M) \simeq \iota^{+} M$ where $\iota$ is given by $\lambda \mapsto-\lambda$, and we set

$$
\hat{\mathcal{M}}_{A}^{\beta}:=\mathrm{FL}^{-1}\left(\mathcal{M}_{A}^{\beta}\right)
$$

with global sections $\hat{M}_{A}^{\beta}$.

Notation 1.6. If $\mathbb{A}^{\ell}$ and $\hat{\mathbb{A}}^{\ell}$ are an FL-pair with $\mathbb{A}^{\ell}=\mathbb{k}^{A}$ for some matrix $A$, we shall denote by $\hat{R}_{A}, \hat{O}_{A}, \hat{S}_{A}, \ldots$ the $A$-graded objects on $\hat{\mathbb{A}}^{\ell}$ corresponding to the $A$-graded objects $R_{A}, O_{A}, S_{A}, \ldots$ on $\mathbb{A}^{\ell}$.

\section{Restriction and De Rham functors of Euler-Koszul Complexes}

In this section we make some computations considering certain functors on the class of (generalized) hypergeometric systems.

2.1. Local cohomology. Relevant in several ways are the local cohomology functors $H_{\partial_{A}}^{\bullet}(-)$ given as the higher derived functors of the $\partial_{A}$-torsion functor

$$
\Gamma_{\partial_{A}}(M):=\left\{m \in M \mid \partial_{i}^{k} \cdot m=0 \forall k \gg 0, \forall i\right\},
$$

a subfunctor of the identity functor on the category of $R_{A}$-modules. If $M$ is $A$ graded, so are all $H_{\partial_{A}}^{i}(M)$ since the support ideal is $A$-graded. See [ILL $\left.{ }^{+} 07\right]$ for details and background.

Lemma 2.1. For any $R_{A}$-module $N$ there is a functorial isomorphism

$$
R \Gamma_{\partial_{A}}(N)\left[\operatorname{dim}\left(X_{A}\right)\right]=\left(D_{A} / \partial_{A} D_{A}\right) \otimes_{R_{A}}^{L} N
$$

so that $H_{\partial_{A}}^{\bullet}(N)=\operatorname{Tor}_{\operatorname{dim}(X)-\bullet}^{R_{A}}\left(D_{A} / \partial_{A} D_{A}, N\right)$. Any $R_{A}$-grading $\operatorname{deg}(-)$ on $N$ makes this isomorphism graded if the right side is shifted by $\sum_{j} \operatorname{deg}\left(\partial_{j}\right)$.

Proof. One representative for $\mathrm{R}_{\partial_{A}}(-)$ is the Čech (i.e., stable Koszul) complex $\check{C}_{A}^{\bullet}(-)=(-) \otimes_{R_{A}} \otimes_{j}\left(R_{A} \longrightarrow R_{A}\left[1 / \partial_{j}\right]\right)$. On $R_{A}$, this returns a $D_{A}$-complex with unique cohomology group, in cohomological degree $\operatorname{dim} X_{A}$, given by $\bigoplus_{\mathbf{v}<0} \mathbb{k} \cdot \partial^{\mathbf{v}}$ where $\mathbf{v}$ is componentwise negative. The $D_{A}$-isomorphism of this module with $D_{A} / D_{A} \partial_{A}$ that identifies the coset of $1 / \prod_{j} \partial_{j}$ in the former with the coset of 1 in the latter (is $A$-graded of degree $\varepsilon_{A}$ and) shows that (up to this shift in degree) this is the injective hull of $R_{A} / R_{A} \cdot \partial_{A}$ over $R_{A}$. The anti-automorphism induced 
by $x^{\mathbf{u}} \partial^{\mathbf{v}} \longrightarrow \partial^{\mathbf{v}}(-x)^{\mathbf{u}}$ allows to view $\check{C}_{A}^{\bullet}$ as complex of right $D_{A}$-modules without affecting the $R_{A}$-structure. Then $H_{\partial_{A}}^{\operatorname{dim}} X_{A}\left(R_{A}\right)=D_{A} / \partial_{A} D_{A}$ is the canonical module of $R_{A}$ with its natural right $D_{A}$-structure.

The modules in $\check{C}_{A}^{\bullet}$ are flat, so $\check{C}_{A}^{\bullet} \otimes_{R_{A}} N\left[\operatorname{dim} X_{A}\right]=D_{A} / \partial_{A} D_{A} \otimes_{R_{A}}^{L} N$. If $N$ is graded, then-since $\partial_{A}$ is monomial $-D_{A} / \partial_{A} D_{A}$ and its flat resolution $\check{C}_{A}^{\bullet}$ are also graded. Hence $\check{C}_{A}^{\bullet} \otimes_{R_{A}} N$ has graded cohomology. The identification $H^{\operatorname{dim} X_{A}}\left(\check{C}_{A}^{\bullet}\right)\left[\operatorname{dim} X_{A}\right] \simeq D_{A} / \partial_{A} D_{A}$ shifts the grading by the degree of the socle element $1 / \prod_{j} \partial_{j}$ of the left hand side.

2.2. Strongly resonant parameters. We recall from [MMW05, SW09] the following important sets. The exceptional locus $\mathcal{E}_{A}$ is

$$
\mathcal{E}_{A}:=\operatorname{qdeg}_{A}\left(\bigoplus_{k>\operatorname{dim} X_{A}-\operatorname{dim} T_{A}} \operatorname{Ext}_{R_{A}}^{k}\left(S_{A}, R_{A}\right)\right)=\bigcup_{k<\operatorname{dim} T_{A}}\left({\overline{\operatorname{deg} H_{\partial_{A}}\left(S_{A}\right)}}^{\text {Zar }}\right) .
$$

A larger interesting set is

$$
\operatorname{sRes}(A):=\bigcup_{j} \operatorname{qdeg}_{A}\left(H_{\partial_{j}}^{1}\left(S_{A}\right)\right),
$$

the strongly resonant parameters of $A$.

For $\mathbb{k}=\mathbb{C}$ the following results were shown in [MMW05, SW09]. A parameter is in $\mathcal{E}_{A}$ if and only if the complex $\mathcal{K}_{\bullet}\left(S_{A} ; E-\beta\right)$ fails to be a resolution of $\mathcal{M}_{A}^{\beta}$; it is in $\operatorname{sRes}(A)$ if and only if $\mathcal{K}_{\bullet}\left(S_{A} ; E-\beta\right)$ fails to resolve the Fourier-Laplace transform of $h_{A+}\left(\mathcal{O}_{T_{A}}^{\beta}\right)$ where

$$
\mathcal{O}_{T_{A}}^{\beta}=\mathcal{D}_{T_{A}} / \mathcal{D}_{T_{A}}\left(\left\{\partial_{t_{i}} t_{i}+\beta_{i}\right\}_{i}\right),
$$

or alternatively if and only if $h_{A+}\left(\mathcal{O}_{T_{A}}^{\beta}\right)$ disagrees with $\hat{\mathcal{M}}_{A}^{\beta}$. We are interested in these results over $\mathbb{k}$ :

Theorem 2.2. Let $\mathbb{k}$ be an arbitrary field of characteristic zero. For each $j$, the following are equivalent:

(1) $\beta \notin \operatorname{sRes}_{j}(A):=\operatorname{qdeg}_{A}\left(H_{\partial_{j}}^{1}\left(S_{A}\right)\right)$;

(2) left-multiplication by $\partial_{x_{j}}$ is a quasi-isomorphism on $K_{\bullet}\left(E-\beta ; S_{A}\right)$.

Corollary 2.3. Over any coefficient field $\mathbb{k}$ of characteristic zero, the following are equivalent:

(1) $\beta \notin \operatorname{sRes}(A)$;

(2) $\mathcal{K}_{\bullet}\left(E-\beta ; S_{A}\right)$ represents the Fourier-Laplace transform of $h_{A+} \mathcal{O}_{T_{A}}^{\beta}$

(3) $\mathcal{M}_{A}^{\beta}$ is naturally isomorphic to the Fourier-Laplace transform of $\mathcal{H}^{0} h_{A+} \mathcal{O}_{T_{A}}^{\beta}$

Inspection shows that, apart from formal computations that do not depend on $\mathbb{k}$, there are the following logical dependencies in [SW09].

- [SW09, Cor. 3.7] needs [SW09, Thm. 3.5, Cor. 3.1,Prop. 2.1] and [MMW05, Prop. 5.3], and the fact that higher Euler-Koszul homology is $\left(\prod_{j} \partial_{j}\right)$ torsion.

- [SW09, Thm. 3.5] needs [SW09, Lem. 3.2] and [MMW05, Prop. 5.3].

- [SW09, Lem. 3.2] is completely formal and independent of the field $\mathbb{k}$.

- [SW09, Cor. 3.1] needs the left and right Øre properties of $D_{A}$, and [SW09, Prop. 2.1]. 
- [SW09, Prop. 2.1] needs that direct images over $\mathbb{k}$ are formally the same for all $\mathbb{k}$ (which they are), plus $\mathcal{D}$-affinity of tori, plus various formal computations contained in $\left[\mathrm{BGK}^{+} 87\right]$, namely an identification of a direct image module in VI.7.3, the chain rule in VI.4.1, exactness of direct images for affine closed embeddings in VI.8.1, and equality of direct images under open embeddings in the $\mathcal{D}$ - and $\mathcal{O}$-category in VI.5.2.

Tori are $\mathcal{D}$-affine since they are $\mathcal{O}$-affine. Higher Euler-Koszul homology is $\left(\prod_{j} \partial_{j}\right)$-torsion since localizing every $\partial_{j}$ leads to Euler-Koszul homology of the quasi-toric Cohen-Macaulay module $\mathbb{k}[\mathbb{Z} A]$ (compare [SW09] for quasi-toricity). The Øre properties of $D_{A}$ rely on the Leibniz rule and are unaffected by $\mathbb{k}$. Any closed embedding over $\mathbb{k}$ can be base-changed to a closed embedding (and hence to an affine faithful map) over $\mathbb{C}$, by viewing $\mathbb{k}$ (algebraically) as a subfield of $\mathbb{C}$. Since $\mathbb{C}$ is fully faithful over $\mathbb{k}$ and affine faithful maps over $\mathbb{C}$ yield exact direct image functors, so do they over $\mathbb{k}$. Direct images for open embeddings agree over $\mathcal{D}$ and $\mathcal{O}$ more or less by definition. It therefore remains to inspect [MMW05, Prop. 5.3] and exactness of the Euler-Koszul complex on maximal Cohen-Macaulay input over $\mathbb{k}$.

Using superscripts to indicate base fields, $K_{A}^{\mathrm{k}}\left(D_{A}^{\mathbb{k}} ; E-\beta\right) \otimes_{\mathrm{k}} \mathbb{C}=K_{A}^{\mathbb{C}}\left(D_{A}^{\mathbb{C}} ; E-\beta\right)$ as long as $\beta \in \mathbb{k} A$. The notion of a toric module is formally independent of $\mathbb{k}$, and so the categories of toric modules and their Euler-Koszul complexes embed into one another for containments of fields. In particular, the formal mechanisms are identical and scale from one field to another faithfully.

The required part of [MMW05, Prop. 5.3] is the equivalence $(3) \Leftrightarrow(4)$. The proof passes through the equivalences $(2) \Leftrightarrow(3)$ and $(2) \Leftrightarrow(4)$. For both we need, modulo formal computations involving toric composition chains, only to check the equivalence of conditions (1) and (3) in [MMW05, Lem. 4.9]. The implication $(1) \Rightarrow(3)$ is linear algebra over any field. The reverse follows by contradiction from base change to $\mathbb{C}$.

Finally, if $M$ is a maximal Cohen-Macaulay toric module over $\mathbb{k}$ then vanishing of higher Euler-Koszul homology follows like over $\mathbb{C}$ from the spectral sequence [MMW05, Thm. 6.3] since the existence of the spectral sequence is abstract homological nonsense. However, this use of the spectral sequence requires the concept of holonomicity: one would like to use that Euler-Koszul homology modules are holonomic and that therefore their duals are modules.

The Euler-Koszul homology modules induce $\mathcal{D}_{A}$-modules on affine space. On that class, (dimension, and hence) holonomicity can be defined over all fields, via the theory of good filtrations. That holonomic modules have holonomic modules as their duals was proved by Roos, see the Bernstein notes [Ber, Thm. 3.15].

2.3. Restriction to the origin. Let $\rho$ be the restriction functor to $\mathbf{0}_{A} \in \mathbb{k}^{A}$,

$$
\rho(-):=\left(D_{A} / x_{A} D_{A}\right) \otimes_{R_{A}}^{L}(-)
$$

from the category of ( $A$-graded) $\mathcal{D}_{A^{-}}$or $D_{A}$-modules to the category of ( $A$-graded) $\mathbb{k}$-vector spaces. Denote $\rho_{k}(-)$ its $k$-th homology.

We start with a topological observation derived from [SW09]. By $H_{d R}^{\bullet}(-; \mathbb{k})$ we mean the algebraic de Rham cohomology in the sense of Grothendieck [Gro66]. 
Lemma 2.4. If $\beta \notin \operatorname{sRes}(A)$ then $\rho\left(\mathcal{M}_{A}^{\beta}\right)$ is naturally identified with the homology of the local system to $\mathcal{O}_{T_{A}}^{\beta}$ on the torus $T_{A}$ :

$$
H_{j}\left(\rho\left(\mathcal{M}_{A}^{\beta}\right)\right)=\rho_{j}\left(\mathcal{M}_{A}^{\beta}\right) \simeq\left\{\begin{array}{cr}
H_{d R}^{j}\left(T_{A} ; \mathbb{k}\right) & \text { if } \beta \in \mathbb{Z} A \backslash \operatorname{sRes}(A) \\
0 & \text { if } \beta \in \mathbb{k} A \backslash(\mathbb{Z} A \cup \operatorname{sRes}(A)) .
\end{array}\right.
$$

Proof. By [SW09] and 2.3, if $\beta \notin \operatorname{sRes}(A)$ then $\mathrm{FL}^{-1}\left(\mathcal{M}_{A}^{\beta}\right) \simeq h_{A+}\left(\mathcal{O}_{T_{A}}^{\beta}\right)$. Under Fourier-Laplace, restriction $\rho$ converts to the functor $\left(D_{A} / \partial_{A} D_{A}\right) \otimes_{D_{A}}^{L_{A}}(-)$. On the affine space $X_{A}=\mathbb{k}^{A}$ this is the $D$-module direct image under the map to a point. Hence with $\beta \notin \operatorname{sRes}(A), \rho\left(\mathcal{M}_{A}^{\beta}\right)$ represents the direct image of $\mathcal{O}_{T_{A}}^{\beta}$ under projection to a point - in other words, the cohomology of the local system.

We next extend this lemma by identifying algebraically $\rho\left(M_{A}^{\beta}\right)$ with $\Lambda(\mathbb{k} A)$ for non-exceptional $\beta$. (We view the exterior algebra as an abstract copy of the cohomology of $T_{A}$ ). Note that in this case the Euler-Koszul complex resolves $\mathcal{M}_{A}^{\beta}$ but is not necessarily a representative for $\mathrm{FL}^{-1} h_{A+}\left(\mathcal{O}_{T_{A}}^{\beta}\right)$. Studying restrictions of Euler-Koszul complexes turns out to be very down to earth.

Lemma 2.5. If $\phi: N \longrightarrow N^{\prime}$ is an A-graded morphism (of degree zero) of A-graded $R_{A}$-modules then

(1) the restriction $\rho\left(\mathcal{K}_{\bullet}(N ; E-\beta)\right)$ is naturally $\Lambda\left(\mathbb{k}_{k} A\right) \otimes_{\mathbb{k}} N_{\beta}$, in the sense that

(2) the induced morphism $\rho\left(\mathcal{K}_{\bullet}(N ; E-\beta)\right) \longrightarrow \rho\left(\mathcal{K}_{\bullet}\left(N^{\prime} ; E-\beta\right)\right)$ is identified with the morphism $\bigwedge(\mathbb{k} A) \otimes_{\mathbb{k}} N_{\beta} \longrightarrow \bigwedge\left(\mathbb{k}_{k} A\right) \otimes_{\mathrm{k}} N_{\beta}^{\prime}$ induced from $\phi_{\beta}$.

Proof. We extend the domain of the Euler-Koszul functor to modules of the form $Q \otimes_{R_{A}} N$ where $N$ is an $A$-graded $R_{A}$-module and $Q$ a right $A$-graded $D_{A}$-module by setting $E_{i} \circ(q \otimes \nu)=q\left(E_{i}+\operatorname{deg}_{A, i}(\nu)\right) \otimes \nu$.

Morally, $E_{i} \circ(-)$ remains right-multiplication by $E_{i}$ and (since multiplications on the left and right commute) one easily checks that there is an isomorphism of functors $\rho\left(\mathcal{K}_{\bullet}(-; E-\beta)\right)=\mathcal{K}_{\bullet}\left(\rho\left(D_{A} \otimes_{R_{A}}(-)\right) ; E-\beta\right)=\mathcal{K}_{\bullet}\left(\left(D_{A} / x_{A} D_{A}\right) \otimes_{R_{A}}^{L}\right.$ $(-) ; E-\beta)$ from the category of $A$-graded $R_{A}$-modules to the category of $A$-graded vector spaces.

As right $R_{A}$-module, $\left(D_{A} / x_{A} D_{A}\right) \otimes_{R_{A}} N=N$ for any $A$-graded $N$. Hence $\left(D_{A} / x_{A} D_{A}\right) \otimes_{R_{A}}^{L}(-)=\left(D_{A} / x_{A} D_{A}\right) \otimes_{R_{A}}(-)$. The $E_{i}$-action is then $E_{i} \circ(1 \otimes \nu)=$ $\left(\operatorname{deg}_{A, i}(\nu)\right) \otimes \nu$. In particular, the Euler-Koszul complex of $E-\beta$ on $\left(D_{A} / x_{A} D_{A}\right) \otimes$ $N$ is in degree $\alpha \in \mathbb{Z} A$ the Koszul complex on $N_{\alpha}$ induced by the numbers $\left\{\alpha_{i}-\beta_{i}\right\}_{i}$. If $\alpha=\beta$ then this Koszul complex is $\bigwedge(\mathbb{k} A) \otimes_{\mathbb{k}} N_{\alpha}$ with zero differential. If $\alpha \neq \beta$ then this Koszul complex is the Koszul complex (over $\mathbb{Z}$ ) of a set of generators of the unit ideal and hence exact.

The final claim is clear from the construction.

Corollary 2.6. If $\beta \notin \mathcal{E}_{A}$ then

$$
\rho_{j}\left(\mathcal{M}_{A}^{\beta}\right) \simeq\left\{\begin{array}{cll}
H_{d R}^{j}\left(T_{A} ; \mathbb{k}\right) & \text { if } & \beta \in \mathbb{N} A \\
0 & \text { if } & \beta \notin \mathbb{N} A
\end{array}\right.
$$

Proof. If $\beta \notin \mathcal{E}_{A}$ then $\rho\left(\mathcal{M}_{A}^{\beta}\right)=\rho\left(\mathcal{K}_{\bullet}\left(S_{A} ; E-\beta\right)\right)$. Now use Lemma 2.5.

The natural morphism $\rho\left(\mathcal{K}_{\bullet}\left(S_{A} ; E-\beta\right)\right) \longrightarrow \rho\left(\mathcal{M}_{A}^{\beta}\right)$ need not be an isomorphism: 
Example 2.7. Let $A=\left(\begin{array}{llll}1 & 1 & 1 & 1 \\ 0 & 1 & 3 & 4\end{array}\right)$ and take $\beta=(1,2)$, the only parameter with higher Euler-Koszul homology for this $A$ (by [ST98]). The $\mathbb{k}$-dimension vectors for $\rho\left(\mathcal{M}_{A}^{\beta}\right)$ and $\rho\left(\mathcal{K}_{\bullet}\left(S_{A} ; E-\beta\right)\right)$ are $(0,0,1,0,0)$ and $(0,0,0,0,0)$ respectively.

In order to better understand the relationship between the restrictions of the $A$-hypergeometric system and the Euler-Koszul complex respectively, we consider the 3-rd quadrant spectral sequence

$$
E_{-i,-j}^{2}=\rho_{j}\left(\mathcal{H}_{i}(-; E-\beta)\right) \Longrightarrow\left(\rho\left(\mathcal{K}_{\bullet}(-; E-\beta)\right)\right)_{i+j} .
$$

The $k$-th differential is $d_{k}: E_{-p,-q}^{k} \longrightarrow E_{-p-k+1,-q+k}^{k}$. A toric map $N \longrightarrow N^{\prime}$ induces a morphism of corresponding spectral sequences.

All our experiments indicate that if $\beta \in \mathbb{N} A$ then $\rho_{j}\left(\mathcal{M}_{A}^{\beta}\right)=H_{d R}^{j}\left(T_{A}\right)$, irrespective of exceptionality. While we cannot show that, we have a one-way estimate:

Lemma 2.8. If $\beta \in \mathbb{N} A$ then there is a natural inclusion $\bigwedge(\mathbb{k} A) \hookrightarrow \rho\left(\mathcal{M}_{A}^{\beta}\right)$.

Proof. Consider the morphism of spectral sequences attached to the inclusion $S_{A} \hookrightarrow$ $\tilde{S}_{A}$ of $S_{A}$ into its normalization. For any $\beta \in \mathbb{N} A$, the induced map of abutments $\rho\left(\mathcal{K}_{\bullet}\left(S_{A} ; E-\beta\right)\right) \longrightarrow \rho\left(\mathcal{K}_{\bullet}\left(\tilde{S}_{A} ; E-\beta\right)\right)$ is an isomorphism by Lemma 2.5. Since $\tilde{S}_{A}$ is Cohen-Macaulay, it has no higher Euler-Koszul homology and so the abutment $\rho\left(\mathcal{K}_{\bullet}\left(\tilde{S}_{A} ; E-\beta\right)\right)$ is stored in the $i=0$ column of the $E^{2}$-term. It follows that the isomorphism on abutments must be coming from the map of the $i=0$ column, for $k \gg 0$. But $E_{0,-j}^{k}$ is a submodule of $E_{0,-j}^{2}$ for $k \geq 2$. In particular, $\rho\left(\mathcal{K}_{\bullet}\left(\tilde{S}_{A} ; E-\beta\right)\right) \simeq \bigwedge\left(\mathbb{k}_{k} A\right)$ is contained in $\rho\left(\mathcal{K}_{\bullet}\left(S_{A} ; E-\beta\right)\right)=\rho\left(\mathcal{M}_{A}^{\beta}\right)$.

2.4. De Rham cohomology. We consider now the effect of $\left(D / \partial_{A} D_{A}\right) \otimes_{D_{A}}^{L}$ (-) on $M_{A}^{\beta}$ and on the Euler-Koszul complex. This behaves differently since $\left(D_{A} / \partial_{A} D_{A}\right) \otimes_{D_{A}} N$ is not $N$ for most $A$-graded $R_{A}$-modules $N$.

Definition 2.9. If $\beta$ is in $\operatorname{deg}_{A}\left(R \Gamma_{\partial_{A}}\left(S_{A}\right)\right)=\bigcup_{k<\operatorname{dim} T_{A}} \operatorname{deg}_{A}\left(H_{\partial_{A}}^{k}\left(S_{A}\right)\right) \subseteq \mathcal{E}_{A}$ it is called strongly A-exceptional.

Theorem 2.10. For any A-graded $R_{A}$-module $N,\left(D / \partial_{A} D_{A}\right) \otimes_{D_{A}}^{L} K_{\bullet}(N ; E-\beta)$ vanishes whenever $\beta$ is not an $A$-degree of $\left.\mathrm{R} \Gamma_{\partial_{A}}(N)\right)$. More precisely,

$$
\left(D / \partial_{A} D_{A}\right) \otimes_{D_{A}}^{L} K_{\bullet}(N ; E-\beta) \simeq\left(\bigoplus_{i} H_{\partial_{A}}^{i}(N)\right)_{\beta} \otimes_{\mathbb{k}} \bigwedge(\mathbb{k} A)\left[\operatorname{dim} X_{A}\right] .
$$

As in Lemma 2.5, an A-graded map $N \longrightarrow N^{\prime}$ induces a map of de Rham complexes that is identified with $\left(\mathrm{R} \Gamma_{\partial_{A}}(N) \longrightarrow \mathrm{R} \Gamma_{\partial_{A}}\left(N^{\prime}\right)\right)_{\beta} \otimes_{\mathbb{k}} \wedge(\mathbb{k} A)\left[\operatorname{dim} X_{A}\right]$.

If $\beta$ is not strongly exceptional (e.g, if $S_{A}$ is Cohen-Macaulay), then

$$
\operatorname{Tor}_{\bullet}^{D_{A}}\left(D / \partial_{A} D_{A}, K_{\bullet}\left(S_{A} ; E-\beta\right)\right)=H_{d R}^{\bullet+\operatorname{dim} X_{A}}\left(T_{A} ; \mathbb{k}\right)
$$

if $\beta$ is in $\operatorname{deg}_{A}\left(H_{\partial_{A}}^{\operatorname{dim} T_{A}}\left(S_{A}\right)\right)$ and zero otherwise.

Proof. As in the proof of Lemma 2.5, we extend the action of the Euler operators to the quotient $\left(D_{A} / \partial_{A} D_{A}\right) \otimes_{R_{A}} N$ for any $A$-graded $N$. Hence $\left(D_{A} / \partial_{A} D_{A}\right) \otimes_{D_{A}}^{L}$ $K_{\bullet}(N ; E-\beta)=K_{\bullet}\left(\left(D_{A} / \partial_{A} D_{A}\right) \otimes_{R_{A}}^{L} N ; E-\beta\right)$ for any $A$-graded $R_{A}$-module $N$.

Recall that $\varepsilon_{A}=\sum_{j} \mathbf{a}_{j}$ and that its components $\varepsilon_{A, i}$ satisfy $E_{i}+\varepsilon_{A, i}=$ $\sum_{j} a_{i j} \partial_{j} x_{j}$. Take now a free $A$-graded $R_{A}$-resolution $F_{\bullet}$ for $N$. Then for any $A$-graded element $P \otimes f \in\left(D_{A} / \partial_{A} D_{A}\right) \otimes_{R_{A}} F_{k}$ the cosets of $\left(E_{i}-\beta_{i}\right) \circ(P \otimes f)$, of 
$\left(E_{i}-\beta_{i}+\operatorname{deg}_{A, i}(P \otimes f)\right) P \otimes f$ and of $\left(-\varepsilon_{A, i}-\beta_{i}+\operatorname{deg}_{A, i}(P \otimes f)\right) P \otimes f$ coincide. So, as in Lemma 2.5, the Euler-Koszul complex on $\left(D_{A} / \partial_{A} D_{A}\right) \otimes F_{\bullet}$ is in degree $\alpha$ the Koszul complex on $D_{A} / \partial_{A} D_{A} \otimes F_{\bullet}$ induced by the numbers $\left\{-\varepsilon_{A_{i}}-\beta_{i}+\alpha_{i}\right\}$. Hence $K \bullet\left(\left(D_{A} / \partial_{A} D_{A}\right) \otimes_{R_{A}}^{L} N ; E-\beta\right)$ can only have cohomology when $\left(D_{A} / \partial_{A} D_{A}\right) \otimes_{R_{A}}^{L} N$ has a cohomology class in degree $\beta+\varepsilon_{A}$. By Lemma 2.1 this is equivalent to $\beta$ being the degree of a nonzero cohomology class in $\check{C}_{A}^{\bullet} \otimes_{R_{A}} N$ which proves the first claim.

If $R \Gamma_{\partial_{A}}(N)$ is non-exact in degree $\beta$ then $\left(D_{A} / \partial_{A} D_{A}\right) \otimes_{R_{A}}^{L}\left(K_{\bullet}\left(N ; E-\beta-\varepsilon_{A}\right)\right)$ is $\left(H_{\partial_{A}}(N)\right)_{\beta}$ tensored with a Koszul complex (shifted by $\left.\operatorname{dim} X_{A}\right)$ on $\operatorname{dim}\left(T_{A}\right)$ maps $\mathbb{k} \longrightarrow \mathbb{k}$ each of which is the zero map. Hence in this case, the resulting cohomology is $\left(H_{\partial_{A}}^{\bullet}(N)\right)_{\beta} \otimes_{\mathbb{k}} \wedge(\mathbb{k} A)\left[\operatorname{dim} X_{A}\right]$. The indicated naturality condition is clear from the discussion.

If $\beta$ is not strongly exceptional then $\left(\mathrm{R} \Gamma_{\partial_{A}}\left(S_{A}\right)\right)_{\beta} \simeq\left(H_{\partial_{A}}^{\operatorname{dim} T_{A}}\left(S_{A}\right)\right)_{\beta}$. The latter is a subquotient of $\mathbb{k}[\mathbb{Z} A]$ and hence its $A$-graded Hilbert function takes values in $\{0,1\}$. The final claim follows.

Example 2.11. Let $A=\left(\begin{array}{llll}1 & 1 & 1 & 1 \\ 0 & 1 & 3 & 4\end{array}\right)$. Then $\left(H_{\partial_{A}}^{2}\left(S_{A}\right)\right)_{\beta}$ is nonzero exactly if $\beta$ is an interior lattice point of $-\mathbb{R}_{+} A$, while $H_{\partial_{A}}^{1}\left(S_{A}\right)$ is a 1-dimensional vector space concentrated in degree $(1,2)$. It follows that $\operatorname{Tor}_{\bullet}^{D_{A}}\left(D / \partial_{A} D_{A}, K_{\bullet}\left(S_{A} ; E-\right.\right.$ $\beta))$ is $H^{\bullet+4}\left(T_{A} ; \mathbb{k}\right)$ when $\beta$ supports $H_{\partial_{A}}^{2}\left(S_{A}\right)$; it is the shifted $H^{\bullet+4}\left(T_{A} ; \mathbb{k}\right)[1]=$ $H^{\bullet+5}\left(T_{A} ; \mathbb{k}\right)$ when $\beta=(1,2)$; it is zero in all other cases.

In particular, no simple general formula (not appealing to local cohomology modules) for $\operatorname{Tor}_{\bullet}^{D_{A}}\left(D / \partial_{A} D_{A}, K_{\bullet}\left(S_{A} ; E-\beta\right)\right)$ comes to mind.

Corollary 2.12. $\operatorname{Tor}_{\bullet}^{D_{A}}\left(D / \partial_{A} D_{A}, M_{A}^{\beta}\right)$ and $\operatorname{Ext}_{D_{A}}^{\bullet}\left(O_{A}, M_{A}^{\beta}\right)$ are nonzero only if

$$
\beta \in \mathcal{E}_{A} \cup\left(\operatorname{deg}_{A}\left(H_{\partial_{A}}^{\operatorname{dim}\left(S_{A}\right)}\left(S_{A}\right)\right)\right) .
$$

Proof. Note first that resolving $O_{A}$ over $D_{A}$ and dualizing the resolution gives a resolution of (a cohomologically shifted) $D_{A} / \partial_{A} D_{A}$, so that the Ext- and Tor-claims are equivalent.

By [MMW05], the Euler-Koszul complex resolves $M_{A}^{\beta}$ whenever $\beta \notin \mathcal{E}_{A}$. So, for such $\beta$ not in $\operatorname{deg}_{A}\left(H_{\partial_{A}}^{\operatorname{dim}\left(S_{A}\right)}\left(S_{A}\right)\right)$, the indicated Ext- and Tor-groups vanish by Theorem 2.10 .

Definition 2.13. Let $N_{A}$ be the interior ideal of $S_{A}$, generated by the monomials whose degrees are in the topological interior of $\mathbb{R}_{+} A$.

Corollary 2.14. If $S_{A}$ is normal, then

$$
\begin{aligned}
\operatorname{Tor}_{\bullet}^{D_{A}}\left(D / \partial_{A} D_{A}, M_{A}^{\beta}\right) & =\operatorname{Tor}_{\bullet}^{D_{A}}\left(D / \partial_{A} D_{A}, K_{\bullet}\left(S_{A} ; E-\beta\right)\right) \\
& =\left\{\begin{array}{cc}
H_{d R}^{\bullet+\operatorname{dim} X_{A}}\left(T_{A} ; \mathbb{k}\right) & \text { if }-\beta \in \operatorname{deg}_{A}\left(N_{A}\right) ; \\
0 & \text { else. }
\end{array}\right.
\end{aligned}
$$

Proof. The exceptional locus is here empty. The interior ideal is the canonical module $\omega_{S_{A}}$ in the $A$-graded category by [BH93, Cor. 6.3.6] while also in the $A$-graded category $\omega_{S_{A}}=\operatorname{Ext}_{R_{A}}^{\operatorname{dim} X_{A}-\operatorname{dim} T_{A}}\left(S_{A}, \omega_{R_{A}}\right)$, [BH93, Prop. 3.6.12]. Then graded local duality [BH93, Thm. 3.6.19] yields that $\operatorname{deg}_{A}\left(H_{\partial_{A}}^{\operatorname{dim} T_{A}}\left(S_{A}\right)\right)=-\operatorname{deg}_{A}\left(N_{A}\right)$. Now use Theorem 2.10. 
For our applications, it is interesting to know that $\mathbb{N} A$ does not meet the strongly $A$-exceptional locus where for $\tau \subseteq A$ a face we write $\partial_{\tau}$ for $\left\{\partial_{j}\right\}_{j \in \tau}$ :

Lemma 2.15. For any face $\tau$ of $A$, no element of $\mathbb{N} A$ is a degree of $\operatorname{R\Gamma }_{\partial_{\tau}}\left(S_{A}\right)$.

Proof. For $j \in \tau, \bigotimes_{\tau}\left(S_{A} \rightarrow S_{A}\left[\partial_{j}^{-1}\right]\right) \simeq\left(S_{A} \rightarrow S_{A}\left[\partial_{j}^{-1}\right]\right) \otimes \bigotimes_{\tau \ni j^{\prime} \neq j}\left(S_{A} \rightarrow\right.$ $\left.S_{A}\left[\partial_{j^{\prime}}^{-1}\right]\right)$. The corresponding double complex spectral sequence starts on the $E^{1}$ page with modules of the form $S_{A}\left[\left(\partial_{j} \cdot \prod_{j^{\prime} \in \tau^{\prime}} \partial_{j^{\prime}}\right)^{-1}\right] / S_{A}\left[\left(\prod_{j^{\prime} \in \tau^{\prime}} \partial_{j^{\prime}}\right)^{-1}\right]$ for all possible $\tau^{\prime} \subseteq \tau \backslash\{j\}$. The $\mathbb{k}_{\mathrm{k}}$-dimension of $A$-graded localizations of $S_{A}$ in each $A$-degree is zero or one, and $S_{A}$ is a domain,. So, $S_{A}\left[\left(\partial_{j} \cdot \prod_{j^{\prime} \in \tau^{\prime}} \partial_{j^{\prime}}\right)^{-1}\right] / S_{A}\left[\left(\prod_{j^{\prime} \in \tau^{\prime}} \partial_{j^{\prime}}\right)^{-1}\right]$ is of dimension zero in each degree $\beta \in \mathbb{N} A$. Hence the same holds for the abutment.

In contrast, elements of $\mathbb{N} A$, including the origin 0 , can indeed be quasi-degrees of lower local cohomology (and hence exceptional parameters):

Example 2.16. Let $A=\left(\begin{array}{ccccc}2 & 1 & 0 & 1 & 0 \\ 0 & 1 & 1 & 0 & 1 \\ 0 & 0 & 0 & 1 & 1\end{array}\right)$. The exceptional locus is the line $\mathbb{k} \cdot \mathbf{a}_{1}$.

The following corollary will be used in Section 3

Corollary 2.17. Suppose $S_{A}$ is Cohen-Macaulay, and put $M=H_{0}\left(S_{A} \partial_{A} ; E-\beta\right)$. Then for $\beta=0$,

$$
\operatorname{Tor}_{i}^{D_{A}}\left(D_{A} / \partial_{A} D_{A}, M\right)=\left\{\begin{array}{cll}
\mathbb{k}^{\operatorname{dim} T_{A}} & \text { if } & i=\operatorname{dim} X_{A} ; \\
\mathbb{k} & \text { if } & i=\operatorname{dim} X_{A}-1 ; \\
0 & \text { if } & i<\operatorname{dim} X_{A}-1 ;
\end{array}\right.
$$

while all Tor-groups vanish if $0 \neq \beta \in \mathbb{N} A$.

Proof. Consider the toric sequence $0 \longrightarrow S_{A} \partial_{A} \longrightarrow S_{A} \longrightarrow \mathbb{k} \longrightarrow 0$. CohenMacaulayness ensures, by [MMW05], that the Euler-Koszul functor produces an exact sequence

$$
0 \longrightarrow H_{1}(\mathbb{k} ; E-\beta) \longrightarrow H_{0}\left(S_{A} \partial_{A} ; E-\beta\right) \longrightarrow M_{A}^{\beta} \longrightarrow H_{0}(\mathbb{k} ; E-\beta) \longrightarrow 0 .
$$

For $\beta \neq 0$, the outer modules are zero. For $\beta=0$, the right module is $O_{A}$ and the left is $O_{A}^{\operatorname{dim}} T_{A}$. The claim then follows from Theorem 2.10 and Lemma 2.15: apply $\operatorname{Tor}_{\bullet}^{D_{A}}\left(D_{A} / \partial_{A} D_{A},-\right)$ to $0 \longrightarrow M / O_{A}^{\operatorname{dim} T_{A}} \longrightarrow M_{A}^{\beta} \longrightarrow O_{A} \longrightarrow 0$ and $0 \longrightarrow O_{A}^{\operatorname{dim} T_{A}} \longrightarrow M \longrightarrow M / O_{A}^{\operatorname{dim} T_{A}} \longrightarrow 0$.

2.5. Ext and the polynomial solution functor. Dualizing a $D_{A}$-resolution of $O_{A}$ gives a resolution of (a cohomologically shifted) $D_{A} / \partial_{A} D_{A}$. Hence, up to shift by $\varepsilon_{A}$ in the $A$-grading, $\operatorname{Ext}_{D_{A}}^{\bullet}\left(O_{A}, M_{A}^{\beta}\right)=\operatorname{Tor}_{\operatorname{dim} X_{A}-\bullet}^{D_{A}}\left(D_{A} / \partial_{A} D_{A}, M_{A}^{\beta}\right)$. In particular, the vanishing results in the previous section apply to $\operatorname{Ext}_{D_{A}}^{\bullet}\left(O_{A}, M_{A}^{\beta}\right)$.

In this subsection we consider the behavior of the solution functor $\operatorname{Hom}_{D_{A}}\left(-, O_{A}\right)$ with values in the ring of polynomials on the class of $A$-hypergeometric modules $M_{A}^{\beta}$. It is immediately clear that $\operatorname{Hom}_{D_{A}}\left(M_{A}^{\beta}, O_{A}\right)$ can only be nonzero if $\beta \in \mathbb{N} A$, and it is an old result that $\beta \in \mathbb{N} A$ implies that $\operatorname{Hom}_{D_{A}}\left(M_{A}^{\beta}, O_{A}\right)$ is 1-dimensional, see [SST00, Prop. 3.4.11]. We investigate here the derived polynomial solution functor and prove 
Theorem 2.18. If $\beta \notin \mathcal{E}_{A}$ (for example, if $S_{A}$ is Cohen-Macaulay) then

$$
\operatorname{Ext}_{D_{A}}^{i}\left(M_{A}^{\beta}, O_{A}\right)=\left\{\begin{array}{cc}
H_{d R}^{i}\left(T_{A} ; \mathbb{k}\right) & \text { if } \quad \beta \in \mathbb{N} A \\
0 & \text { else. }
\end{array}\right.
$$

(All experiments indicate this to be true even if $\beta \in \mathcal{E}_{A}$.)

Proof. Write $\tau(-)$ for the transposition $x^{\mathbf{u}} \partial^{\mathbf{v}} \mapsto \partial^{\mathbf{v}}(-x)^{\mathbf{u}}$ on $D_{A}$. Let $F_{\bullet}$ be an $A$-graded $R_{A}$-free resolution of $S_{A}$ and denote $\omega_{R_{A}}=D_{A} / \partial_{A} D_{A}\left[\operatorname{dim} X_{A}\right]$. Then we have the following equalities, where $(-)^{\vee}$ is the vector space dual:

$$
\begin{aligned}
\left.\left(\mathrm{RHom}_{D_{A}}\left(M_{A}^{\beta}, O_{A}\right)\right)\right)^{\vee} & \stackrel{(\mathrm{a})}{\simeq} \mathrm{R} \operatorname{Hom}_{D_{A}}\left(O_{A}, \mathbb{D} M_{A}^{\beta}\right) \\
& \stackrel{(\mathrm{b})}{\simeq} \omega_{R_{A}} \otimes_{D_{A}}^{L} \mathbb{D} M_{A}^{\beta} \\
& \stackrel{(\mathrm{c})}{=} \omega_{R_{A}} \otimes_{D_{A}}^{L} \mathbb{D} K_{\bullet}\left(S_{A} ; E-\beta\right) \\
& \stackrel{(\mathrm{d})}{=} \omega_{R_{A}} \otimes_{D_{A}}^{L} \mathbb{D} K_{\bullet}\left(F_{\bullet} ; E-\beta\right) \\
& \stackrel{(\mathrm{e})}{=} \omega_{R_{A}} \otimes_{D_{A}}^{L} K_{\bullet}\left(\operatorname{Hom}_{R_{A}}\left(F_{\bullet}, R_{A}\right) ; E+\beta+\varepsilon_{A}\right) \\
& \simeq \omega_{R_{A}} \otimes_{D_{A}}^{L} K \bullet\left(\operatorname{RHom}_{R_{A}}\left(S_{A}, R_{A}\right) ; E+\beta+\varepsilon_{A}\right) \\
& \stackrel{(\mathrm{f})}{\simeq} K_{\bullet}\left(\omega_{R_{A}} \otimes_{R_{A}}^{L} \mathrm{R} \operatorname{Hom}_{R_{A}}\left(S_{A}, R_{A}\right) ; E+\beta+\varepsilon_{A}\right) \\
& \stackrel{(\mathrm{g})}{\simeq}\left(\mathrm{R}_{\partial_{A}} \mathrm{R} \operatorname{Hom}_{R_{A}}\left(S_{A}, R_{A}\right)\right)_{-\beta-\varepsilon_{A}} \otimes \bigwedge(\mathbb{k} A) \\
& \stackrel{(\mathrm{h})}{\simeq}\left(\left(\mathrm{R} \operatorname{Hom}_{R_{A}}\left(\mathrm{RHom} \operatorname{Hom}_{R_{A}}\left(S_{A}, R_{A}\right), R_{A}\right)\right)_{\beta} \otimes \bigwedge(\mathbb{k} A)\right)^{\vee} \\
& =\left(\left(S_{A}\right)_{\beta} \otimes \bigwedge(\mathbb{k} A)\right)^{\vee} .
\end{aligned}
$$

The following notes justify the above transformations:

-(a) Duality gives $\mathrm{R} \mathrm{Hom}_{D_{A}}\left(M, M^{\prime}\right) \simeq\left(\mathrm{R} \operatorname{Hom}_{D_{A}}\left(\mathbb{D} M^{\prime}, \mathbb{D} M\right)\right)^{\vee}$, [HTT08, §2.6].

-(b) Resolve $O_{A}$ and dualize the resolution, incurring a cohomological shift.

-(c) By [MMW05], the hypergeometric system is resolved by the Euler-Koszul complex as long as $\beta$ is not exceptional.

-(d) The Euler-Koszul functor can be applied to any $A$-graded resolution.

-(e) $K_{\bullet}\left(F_{\bullet} ; E-\beta\right)$ is a free complex. Applying $\operatorname{Hom}_{D_{A}}\left(-, D_{A}\right)$ and the transposition $\tau$ turns $D_{A} \otimes_{R_{A}} F_{\bullet}$ into $D_{A} \otimes_{R_{A}} \operatorname{Hom}_{R_{A}}\left(F_{\bullet}, R_{A}\right)$ and the EulerKoszul complex on $\beta$ into that on $-\beta-\varepsilon_{A}$ since $x_{j} \partial_{j}$ turns into $-\partial_{j} x_{j}$.

-(f) As in the proof of Theorem 2.10.

-(g) Theorem 2.10 works for $A$-graded complexes just as well.

-(h) Apply local $A$-graded duality (responsible for the dual).

\section{ThreE FOUR-TERM SEQUENCES}

Notation. From now on, $A$ is a $(d+1) \times(n+1)$ matrix and $\mathbb{N} A$ is assumed to be saturated, in addition to the conventions in Notation 1.2 and Definition 1.3.

All products of $\mathbb{k}$-schemes are by default over $\mathbb{k}$.

Consider the exact toric sequence $0 \longrightarrow S_{A} \partial_{A} \longrightarrow S_{A} \longrightarrow \mathbb{k} \longrightarrow 0$. Normality ensures, by [MMW05, Prop. 5.3, Thm. 6.6], that the Euler-Koszul functor produces 
the exact sequence (2.4.1), and, for $i \geq 1$, isomorphisms

$$
H_{i}(\mathbb{k} ; E-\beta) \simeq \begin{cases}O_{A}^{\left(\begin{array}{c}
d+1 \\
i
\end{array}\right)} & \text { for } \beta=0 \\
0 & \text { else }\end{cases}
$$

In this section we will show that the sequence (2.4.1) has a geometric interpretation when $A$ is homogeneous. Our approach is inspired by [Sti98], where Stienstra defined on the torus $T_{A}$ a family $F$ of Laurent polynomials using the matrix $A$. He showed that one term in the long exact cohomology sequence of the pair $\left(T_{A}\right.$, fiber of $F$ ) could be naturally identified with a fiber in the $A$-hypergeometric system $M_{A}^{0}$ when $F$ is smooth. We will extend this identification to the non-smooth fibers of $F$.

We will proceed as follows. First we identify the second term of (2.4.1) as a concatenation of (proper) direct image functors applied to the structure sheaf $\mathcal{O}_{T_{A}}$. The third term already has such an interpretation by Corollary 2.3 above. The remaining terms are identified as the cohomology of the cone of a natural adjunction morphism between the second and third term.

As a second step we show in Lemma 3.7 that the sequence (2.4.1) is part of a long exact sequence coming from a triangle of elementary $\mathcal{D}$-modules on the line $\hat{\mathbb{A}}^{1}$. We also show in Proposition 3.8 that the Fourier-Laplace transform of (2.4.1) is induced by the FL-transformed triangle of elementary $\mathcal{D}$-modules from Lemma 3.7. This enables us to give a geometric interpretation of the exact sequence in Theorem 3.10 in terms of Gauß-Manin systems of the pair $\left(T_{A}\right.$, fiber of $F$ ) as alluded to above.

As a preparatory result we begin with an identification of two functors on certain sheaves.

3.1. Quasi-equivariant bundles. Denote $\mathbb{G}_{m}$ the scheme of units of $\mathbb{k}$. A $\mathbb{G}_{m^{-}}$ action on the variety $Y$ is a multiplicative morphism $\mu: \mathbb{G}_{m} \times Y \longrightarrow Y$ where $1 \in \mathbb{G}_{m}$ acts as identity. That is, $\mu$ is a morphism, $\mu\left(g, \mu\left(g^{\prime}, y\right)\right)=\mu\left(g g^{\prime}, y\right)$ and $\mu(1, y)=y$.

Let $X$ be an affine smooth variety and $\pi: E=X \times \mathbb{A}^{n} \rightarrow X$ be a trivial vector bundle on $X$. Write $E^{*}=E \backslash(X \times\{0\})$ and let $E_{x}$ be the fiber over $x \in X$. The zero section is identified with $X$ as closed subvariety via the embedding

$$
i: X \hookrightarrow E \text {. }
$$

Definition 3.1. A $\mathbb{G}_{m}$-action $\mu: \mathbb{G}_{m} \times E \longrightarrow E$ on $E$ is fibered if

(1) $\mu$ preserves fibers, $\mu: \mathbb{G}_{m} \times E_{x} \longrightarrow E_{x}$;

(2) $\mu$ is the restriction of a morphism $\mu: \mathbb{A}^{1} \times E \longrightarrow E$ under $\mathbb{G}_{m} \hookrightarrow \mathbb{A}^{1}$;

(3) $0 \in \mathbb{A}^{1}$ multiplies into the zero section, $\mu: 0 \times E_{x} \longrightarrow i(X)$;

(4) $\mathbb{A}^{1}$ fixes the zero section, $\mu: \mathbb{A}^{1} \times i(X) \longrightarrow i(X)$.

Definition 3.2. Let $\mu: \mathbb{G}_{m} \times E \rightarrow E$ be a fibered $\mathbb{G}_{m}$-action on $E$. Write $p: \mathbb{G}_{m} \times E \longrightarrow E$ for the projection and denote by $\mu^{\prime}$ and $p^{\prime}$ the restrictions of $\mu$ and $p$ to $\mathbb{G}_{m} \times E^{*}$.

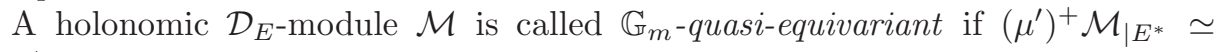
$\left(p^{\prime}\right)^{+} \mathcal{M}_{\mid E^{*}}$

We consider the derived category of bounded complexes of $\mathcal{D}_{E}$-modules with holonomic and quasi-equivariant cohomology. 
Lemma 3.3. Let $\pi: E \rightarrow X$ be fibered and denote $i: X \rightarrow E$ the inclusion of the zero section. For every $\mathbb{G}_{m}$-quasi-equivariant $\mathcal{D}_{E}$-module $\mathcal{M}$,

$$
\pi_{+} \mathcal{M} \simeq i^{\dagger} \mathcal{M} \quad \text { and } \quad \pi_{+} \mathcal{M} \simeq i^{+} \mathcal{M}
$$

Proof. By duality, it suffices to prove the first claim. Denote by $j: E^{*} \rightarrow E$ the open embedding of the complement of the zero section and let $\pi$ be the projection to the base $X$. We have the exact triangles

$$
\begin{gathered}
j_{\dagger} j^{-1} \mathcal{M} \longrightarrow \mathcal{M} \longrightarrow i_{+} i^{\dagger} \mathcal{M} \stackrel{+1}{\longrightarrow} \\
\pi_{+} j_{\dagger} j^{-1} \mathcal{M} \longrightarrow \pi_{+} \mathcal{M} \longrightarrow i^{\dagger} \mathcal{M} \stackrel{+1}{\longrightarrow}
\end{gathered}
$$

and the Cartesian diagram

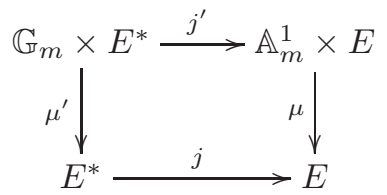

where $\mu^{\prime}$ is the restriction of $\mu$ to $\mathbb{G}_{m} \times E^{*}$ and $j^{\prime}$ is the canonical inclusion. The morphism $s: E \rightarrow \mathbb{A}^{1} \times E$ with $s(x)=(1, x)$ is a section of $\mu$. Thus, the composition (induced by the natural transformation $\operatorname{id}_{E} \longrightarrow \mu_{+} \mu^{\dagger}$ )

$$
\pi_{+} j_{\dagger} j^{-1} \mathcal{M} \rightarrow \pi_{+} \mu_{+} \mu^{\dagger} j_{\dagger} j^{-1} \mathcal{M} \rightarrow \pi_{+} \mu_{+} s_{+} s^{\dagger} \mu^{\dagger} j_{\dagger} j^{-1} \mathcal{M}=\pi_{+} j_{\dagger} j^{-1} \mathcal{M}
$$

is an isomorphism by (3.1.2); hence it is enough to prove $\pi_{+} \mu_{+} \mu^{\dagger} j_{\dagger} j^{-1} \mathcal{M}=0$. By base change,

$$
\pi_{+} \mu_{+} \mu^{\dagger} j_{\dagger} j^{-1} \mathcal{M} \simeq \pi_{+} \mu_{+} j_{\dagger}^{\prime}\left(\mu^{\prime}\right)^{\dagger} j^{-1} \mathcal{M}
$$

Since $\mathcal{M}$ is $\mathbb{G}_{m}$-quasi-equivariant, we have

$$
\left(\mu^{\prime}\right)^{\dagger} j^{-1} \mathcal{M} \simeq p^{\dagger} j^{-1} \mathcal{M} \simeq \mathcal{O}_{\mathbb{G}_{m}} \otimes j^{-1} \mathcal{M} .
$$

Therefore (letting $a: \mathbb{A}^{1} \rightarrow\{p t\}$ be the map to the point) we get

$$
\begin{aligned}
\pi_{+} \mu_{+} j_{\dagger}^{\prime}\left(\mu^{\prime}\right)^{\dagger} j^{-1} \mathcal{M} & \simeq \pi_{+} \mu_{+} j_{\dagger}^{\prime}\left(\mathcal{O}_{\mathbb{G}_{m}} \otimes j^{-1} \mathcal{M}\right) \\
& \simeq \pi_{+} \mu_{+}\left(j_{1 \dagger} \mathcal{O}_{\mathbb{G}_{m}} \otimes j_{\dagger} j^{-1} \mathcal{M}\right) \\
& \simeq \pi_{+} p_{+}\left(j_{1 \dagger} \mathcal{O}_{\mathbb{G}_{m}} \otimes j_{\dagger} j^{-1} \mathcal{M}\right) \\
& \simeq a_{+} j_{1 \dagger} \mathcal{O}_{\mathbb{G}_{m}} \otimes \pi_{+} j_{\dagger} j^{-1} \mathcal{M}
\end{aligned}
$$

where $j_{1}: \mathbb{G}_{m} \rightarrow \mathbb{A}^{1}$ is the canonical inclusion. Since $a_{+} j_{1 \dagger} \mathcal{O}_{\mathbb{G}_{m}}=0$ in $\mathrm{D}^{b}\left(\mathcal{D}_{\{p t\}}\right)$ we have $\pi_{+} \mu_{+} \mu^{\dagger} j_{\dagger} j^{-1} \mathcal{M}=0$.

Recall that $A$ is a $(d+1) \times(n+1)$ matrix. Let $T_{A}=\operatorname{Spec}\left(\mathbb{k}\left[t_{0}^{ \pm}, \ldots, t_{d}^{ \pm}\right]\right)$and consider the ring homomorphism

$$
\begin{aligned}
\mathbb{k}\left[y_{0}, \ldots, y_{n}\right] & \longrightarrow \mathbb{k}\left[t_{0}^{ \pm}, \ldots, t_{d}^{ \pm}\right] \\
y_{i} & \mapsto t^{\mathbf{a}_{i}}
\end{aligned}
$$

which gives rise to a morphism

$$
h_{A}: T_{A} \rightarrow \hat{\mathbb{A}}^{n+1},
$$

where $\hat{\mathbb{A}}^{n+1}=\operatorname{Spec}\left(\mathbb{k}_{k}\left[y_{0}, \ldots, y_{n}\right]\right)$. We factorize this embedding as

$$
T_{A} \stackrel{h_{1}}{\longrightarrow} \hat{\mathbb{A}}^{n+1} \backslash\{0\} \stackrel{h_{2}}{\longrightarrow} \hat{\mathbb{A}}^{n+1} .
$$


We are now ready to show a useful property of $A$-hypergeometric systems and their Fourier-Laplace transforms.

Lemma 3.4. The $\mathcal{D}_{\hat{\mathbb{A}}^{n+1}}$-module $h_{A+} \mathcal{O}_{T_{A}}$ is $\mathbb{G}_{m}$-quasi-equivariant.

Proof. We view $\hat{A}^{n+1}$ as trivial bundle over itself. Since $A$ is pointed, there is $\mathbf{u} \in \mathbb{Z}^{d+1}$ with $\mathbf{v}=\mathbf{u}^{T} \cdot A$ componentwise positive. Let $\mu^{\prime}: \mathbb{G}_{m} \times \hat{\mathbb{A}}^{n+1} \longrightarrow \hat{\mathbb{A}}^{n+1}$ be the monomial action induced by $\mathbf{v}$ and let $\tilde{\mu}: \mathbb{G}_{m} \times T_{A} \longrightarrow T_{A}$ be the action induced by $\mathbf{u}$. (Compare the discussion on the Euler space in [RSW17].) Consider the Cartesian diagram

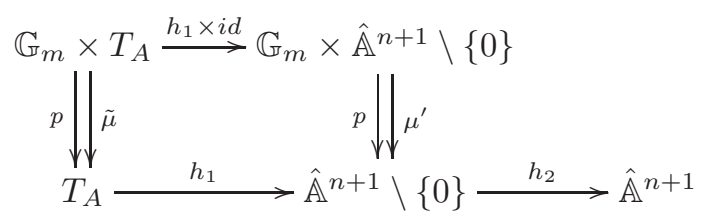

and note that the positivity of $\mathbf{v}$ allows to extend $\mu^{\prime}$ to $\mathbb{A}^{1} \times \hat{\mathbb{A}}^{n+1}$. Then

$$
p^{+} h_{1+} \mathcal{O}_{T_{A}} \simeq\left(h_{1} \times i d\right)_{+} p^{+} \mathcal{O}_{T_{A}} \simeq\left(h_{1} \times i d\right)_{+} \tilde{\mu}^{+} \mathcal{O}_{T_{A}} \simeq\left(\mu^{\prime}\right)^{+} h_{1+} \mathcal{O}_{T_{A}}
$$

and so $p^{+} h_{1+} \mathcal{O}_{T_{A}} \simeq\left(\mu^{\prime}\right)^{+} h_{1+} \mathcal{O}_{T_{A}}$.

3.2. The four-term sequence in terms of direct images. Recall Notation 1.6 regarding Fourier-Laplace transforms and consider the inverse Fourier-Laplace transformation of the sequence (2.4.1):

$$
0 \longrightarrow H_{1}(\mathbb{k} ; \hat{E}+\beta) \longrightarrow H_{0}\left(\hat{S}_{A} \cdot y_{A} ; \hat{E}+\beta\right) \longrightarrow \hat{M}_{A}^{\beta} \longrightarrow H_{0}(\mathbb{k} ; \hat{E}+\beta) \longrightarrow 0 .
$$

Definition 3.5. Let $\mathcal{B}_{0}$ be the unique simple $\mathcal{D}_{\hat{\mathbb{A}}^{n+1}}$-module supported in $0 \in \hat{\mathbb{A}}^{n+1}$. $\diamond$

Proposition 3.6. For $\beta=0$ there is an isomorphism of exact 4-term sequences

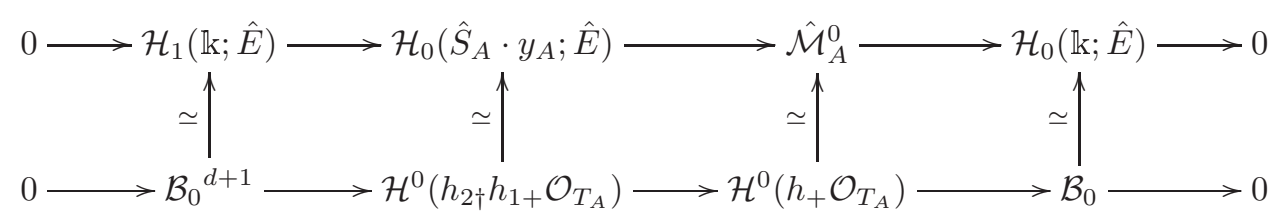

Proof. By Corollary 2.3, $h_{A+} \mathcal{O}_{T_{A}} \simeq \hat{\mathcal{M}}_{A}^{0}$. By $\left.(3.0 .1), \mathcal{H}_{i}(\mathbb{k} ; \hat{E}) \simeq \mathcal{B}_{0}{ }^{\left({ }^{d+1}\right.}\right)$. Restricting to $\hat{\mathbb{A}}^{n+1} \backslash\{0\}$ we see that

$$
\mathcal{H}_{0}\left(\hat{S}_{A} \cdot y_{A} ; \hat{E}\right)_{\mid \hat{\mathbb{A}}^{n+1} \backslash\{0\}} \simeq\left(\hat{\mathcal{M}}_{A}^{0}\right)_{\mid \hat{\mathbb{A}}^{n+1} \backslash\{0\}} \simeq h_{1+} \mathcal{O}_{T_{A}} \quad \text { in } \quad \operatorname{Mod}_{h}\left(\mathcal{D}_{\hat{\mathbb{A}}^{n+1}}\right) .
$$

Since $\mathcal{H}_{>0}\left(S_{A} \cdot y_{A} ; \hat{E}\right)_{\mid \hat{\mathbb{A}}^{n+1} \backslash\{0\}}=0$, we have

$$
\mathcal{H}_{0}\left(S_{A} \cdot y_{A} ; \hat{E}\right)_{\mid \hat{\mathbb{A}}^{n+1} \backslash\{0\}} \simeq \mathcal{K}_{\bullet}\left(S_{A} \cdot y_{A} ; \hat{E}\right)_{\mid \hat{\mathbb{A}}^{n+1} \backslash\{0\}} \quad \text { in } \quad D_{h}^{b}\left(\mathcal{D}_{\hat{\mathbb{A}}^{n+1}}\right) .
$$

By adjunction this gives a morphism

$$
h_{2 \dagger} h_{1+} \mathcal{O}_{T_{A}} \stackrel{\simeq}{\longrightarrow} h_{2 \dagger} h_{2}^{-1} \mathcal{K}_{\bullet}\left(\hat{S}_{A} \cdot y_{A} ; \hat{E}\right) \longrightarrow \mathcal{K}_{\bullet}\left(\hat{S}_{A} \cdot y_{A} ; \hat{E}\right)
$$

and so induces a morphism $\mathcal{H}^{0}\left(h_{2 \dagger} h_{1+} \mathcal{O}_{T_{A}}\right) \longrightarrow \mathcal{H}_{0}\left(\hat{S}_{A} \cdot y_{A} ; \hat{E}\right)$ such that the center and right squares in our diagram commute. We need to prove that the morphism

$$
h_{2 \dagger} h_{2}^{-1} \mathcal{K}_{\bullet}\left(\hat{S}_{A} \cdot y_{A} ; \hat{E}\right) \longrightarrow \mathcal{K}_{\bullet}\left(\hat{S}_{A} \cdot y_{A} ; \hat{E}\right)
$$


is an isomorphism. (While we know that $\mathcal{H}_{1}(\mathbb{k} ; \hat{E})$ and $\mathcal{B}_{0}{ }^{d+1}$ are isomorphic, it is not yet clear that $\mathcal{H}^{0}\left(h_{2 \dagger} h_{1+} \mathcal{O}_{T_{A}}\right) \rightarrow \mathcal{H}_{0}\left(\hat{S}_{A} \cdot y_{A} ; \hat{E}\right)$ induces such isomorphism.)

In order to prove that the morphism (3.2.2) is an isomorphism we have to show that the third term in the adjunction triangle

$$
h_{2 \dagger} h_{2}^{-1} \mathcal{K}_{\bullet}\left(\hat{S}_{A} \cdot y_{A} ; \hat{E}\right) \longrightarrow \mathcal{K}_{\bullet}\left(\hat{S}_{A} \cdot y_{A} ; \hat{E}\right) \longrightarrow i_{+} i^{\dagger} \mathcal{K}_{\bullet}\left(\hat{S}_{A} \cdot y_{A} ; \hat{E}\right) \stackrel{+1}{\longrightarrow}
$$

vanishes. By Kashiwara equivalence it is enough to show that $i^{\dagger} \mathcal{K}_{\bullet} \cdot\left(\hat{S}_{A} \cdot y_{A} ; \hat{E}\right)$ is isomorphic to zero. Since $\mathcal{K}_{\bullet}\left(\widehat{S}_{A} \cdot y_{A} ; \hat{E}\right)_{\mid \hat{\mathbb{A}}^{n+1} \backslash\{0\}} \simeq\left(h_{A+} \mathcal{O}_{T}\right)_{\mid \hat{\mathbb{A}}^{n+1} \backslash\{0\}}$, the complex $\mathcal{K}_{\bullet}\left(\widehat{S}_{A} \cdot y_{A} ; \hat{E}\right)$ is $\mathbb{G}_{m}$-quasi-equivariant. By Lemma $3.3, i^{\dagger} \mathcal{K}_{\bullet}\left(\widehat{S}_{A} \cdot y_{A} ; \hat{E}\right) \simeq$ $a_{+} \mathcal{K}_{\bullet}\left(\widehat{S}_{A} \cdot y_{A} ; \hat{E}\right)$ where $a$ is the map to a point. Now $a_{+} \mathcal{K}_{\bullet}\left(\widehat{S}_{A} \cdot y_{A} ; \hat{E}\right)$ is dual to $\rho \mathcal{K}_{\bullet}\left(S_{A} \partial_{A} ; E\right)$ which allows us to use Lemma 2.5 to conclude.

\subsection{The four-term sequence with Gauß-Manin systems.}

Notation. From now on, in addition to the assumptions in Notation 1.2 and Definition 1.3 as well as normality, we assume that the matrix $A$ is homogeneous, i.e. that $(1, \ldots, 1)$ is in the row span of $A$.

Furthermore, for the remainder of the paper, $\beta=0$.

One may put $A$ into the following shape by elementary row operations

$$
A=\left(\mathbf{a}_{1}, \ldots, \mathbf{a}_{n}\right)=\left(\begin{array}{cccc}
1 & 1 & \ldots & 1 \\
0 & a_{11} & \ldots & a_{1 n} \\
\vdots & \vdots & & \vdots \\
0 & a_{d 1} & \ldots & a_{d n}
\end{array}\right)=\left(\begin{array}{cccc}
1 & 1 & \ldots & 1 \\
0 & & & \\
\vdots & & B & \\
0 & &
\end{array}\right)
$$

where $B=\left(\mathbf{b}_{1}, \ldots, \mathbf{b}_{n}\right)$ is the $d \times n$-matrix with entries $\left(a_{i j}\right)_{1 \leq i \leq d, 1 \leq j \leq n}$.

Using this homogeneity assumption, we will here give a geometric interpretation to our 4-term sequence (2.4.1). For this we will need a variant of a comparison theorem of d'Agnolo and Eastwood [DE03], between the Radon and Fourier-Laplace transform, and several other preparatory statements.

Set $T_{B}:=\operatorname{Spec}\left(\mathbb{k}\left[t_{1}^{ \pm}, \ldots, t_{d}^{ \pm}\right]\right)$and $\hat{\mathbb{A}}^{1}:=\operatorname{Spec}\left(\mathbb{k}\left[t_{0}\right]\right)$. We will identify $T_{A}$ with $T_{B} \times\left(\hat{\mathbb{A}}^{1} \backslash\{0\}\right)$. From the ring homomorphism

$$
\begin{aligned}
\mathbb{k}\left[y_{0}, \ldots, y_{n}\right] & \longrightarrow \mathbb{k}\left[t_{0}, t_{1}^{ \pm}, \ldots, t_{d}^{ \pm}\right] \\
\left(y_{0}, \ldots, y_{n}\right) & \mapsto\left(t_{0}, t_{0} t^{\mathbf{b}_{1}}, \ldots, t_{0} t^{\mathbf{b}_{n}}\right)
\end{aligned}
$$

we get a map

$$
k: T_{B} \times \hat{\mathbb{A}}^{1} \longrightarrow \hat{\mathbb{A}}^{n+1}
$$

whose restriction to $T_{A}$ is just our old morphism $h_{A}$. Let $\tilde{k}$ be the closed embedding

$$
\tilde{k}:=\left(i d_{T_{B}} \times k\right): T_{B} \times \hat{\mathbb{A}}^{1} \rightarrow T_{B} \times \hat{\mathbb{A}}^{n+1},
$$

let $j, i$ be the embedding and inclusion

$$
j: T_{A}=T_{B} \times\left(\hat{\mathbb{A}}^{1} \backslash\{0\}\right) \rightarrow T_{B} \times \hat{\mathbb{A}}^{1}, \quad i: T_{B} \times\{0\} \rightarrow T_{B} \times \hat{\mathbb{A}}^{1} .
$$


Then there is a commutative diagram

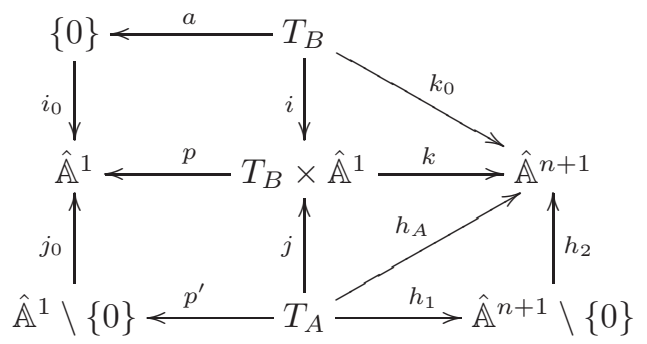

where $p: T_{B} \times \hat{\mathbb{A}}^{1} \rightarrow \hat{\mathbb{A}}^{1}$ is the projection and $k_{0}$ sends $T_{B}$ to the origin. Define the following $\mathcal{D}$-modules on $\hat{\mathbb{A}}^{1}$ :

$$
\begin{array}{lr}
\mathcal{D}_{\widehat{\mathbb{A}}^{1}} \bullet 1:=\mathcal{D}_{\hat{\mathbb{A}}^{1}} /\left(\partial_{t}\right), \quad \mathcal{D}_{\hat{\mathbb{A}}^{1}} \bullet \mathfrak{H}:=\mathcal{D}_{\hat{\mathbb{A}}^{1}} /\left(t \partial_{t}\right), \\
\mathcal{D}_{\widehat{\mathbb{A}}^{1}} \bullet 1 / t:=\mathcal{D}_{\hat{\mathbb{A}}^{1}} /\left(\partial_{t} t\right), \quad \mathcal{D}_{\hat{\mathbb{A}}^{1}} \bullet \delta:=\mathcal{D}_{\hat{\mathbb{A}}^{1}} /(t) .
\end{array}
$$

(The module $\mathcal{D}_{\widehat{\mathbb{A}}^{1}} \bullet \mathfrak{H}$ encodes the Heaviside distribution).

Lemma 3.7. We have the following isomorphisms:

$$
\begin{aligned}
k_{+} \mathcal{O}_{T_{B} \times \hat{\mathbb{A}}^{1}} & \simeq k_{+} p^{+}\left(\mathcal{D}_{\hat{\mathbb{A}}^{1}} \bullet 1\right), & h_{A+} \mathcal{O}_{T_{A}} & \simeq k_{+} p^{+}\left(\mathcal{D}_{\hat{\mathbb{A}}^{1}} \bullet 1 / t\right), \\
k_{0+} \mathcal{O}_{T_{B}} & \simeq k_{+} p^{+}\left(\mathcal{D}_{\hat{\mathbb{A}}^{1}} \bullet \delta\right), & h_{2 \dagger} h_{1+} \mathcal{O}_{T_{A}} & \simeq k_{+} p^{+}\left(\mathcal{D}_{\hat{\mathbb{A}}^{1}} \bullet \mathfrak{H}\right) .
\end{aligned}
$$

The adjunction morphism $h_{2 \dagger} h_{1+} \mathcal{O}_{T_{A}} \rightarrow h_{A+} \mathcal{O}_{T_{A}}$ is induced by the adjunction morphism $\mathcal{D}_{\hat{\mathbb{A}}^{1}} \bullet \mathfrak{H} \rightarrow \mathcal{D}_{\hat{\mathbb{A}}^{1}} \bullet 1 / t$.

Proof. The first three isomorphisms follow from

$$
\begin{aligned}
& k_{+} \mathcal{O}_{T_{B} \times \hat{\mathbb{A}}^{1}} \simeq k_{+} p^{+} \mathcal{O}_{\hat{\mathbb{A}}^{1}}=k_{+} p^{+}\left(\mathcal{D}_{\hat{\mathbb{A}}^{1}} \bullet 1\right), \\
& h_{A+} \mathcal{O}_{T_{A}} \simeq k_{+} j_{+} \mathcal{O}_{T_{A}} \simeq k_{+} j_{+} p^{\prime+} \mathcal{O}_{\hat{\mathbb{A}}^{1} \backslash\{0\}} \simeq k_{+} p^{+} j_{0+} \mathcal{O}_{\hat{\mathbb{A}}^{1} \backslash\{0\}} \simeq k_{+} p^{+}\left(\mathcal{D}_{\hat{\mathbb{A}}^{1}} \bullet 1 / t\right), \\
& k_{0+} \mathcal{O}_{T_{B}} \simeq k_{+} i_{+} \mathcal{O}_{T_{B}} \simeq k_{+} i_{+} a^{+} \mathcal{O}_{\{0\}} \simeq k_{+} p^{+} i_{0} \mathcal{O}_{\{0\}} \simeq k_{+} p^{+}\left(\mathcal{D}_{\hat{\mathbb{A}}^{1}} \bullet \delta\right) .
\end{aligned}
$$

For the last one we have

$$
k_{+} p^{+}\left(\mathcal{D}_{\hat{\mathbb{A}}^{1}} \bullet \mathfrak{H}\right) \simeq k_{+} p^{+} j_{0 \dagger} \mathcal{O}_{\hat{\mathbb{A}}^{1} \backslash\{0\}} \simeq k_{+} j_{\dagger} p^{\prime+} \mathcal{O}_{\hat{\mathbb{A}}^{1} \backslash\{0\}} \simeq k_{+} j_{\dagger} \mathcal{O}_{T_{A}} .
$$

So it remains to prove that $k_{+} j_{\dagger} \mathcal{O}_{T_{A}} \simeq h_{2 \dagger} h_{1+} \mathcal{O}_{T_{A}}$. For this consider the diagram with Cartesian squares

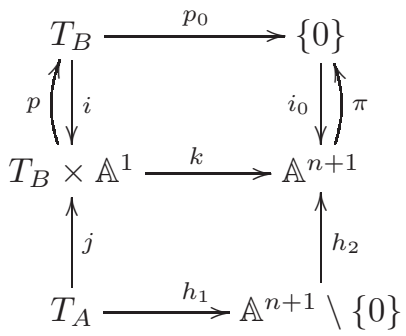

Base change in the lower square gives $h_{1+} \mathcal{O}_{T_{A}} \stackrel{\simeq}{\rightarrow} h_{1+} j^{-1} j_{\dagger} \mathcal{O}_{T_{A}} \stackrel{\simeq}{\rightrightarrows} h_{2}^{-1} k_{+} j_{\dagger} \mathcal{O}_{T_{A}}$. Adjunction yields a morphism $h_{2 \dagger} h_{1+} \mathcal{O}_{T_{A}} \rightarrow k_{+} j_{\dagger} \mathcal{O}_{T_{A}}$. In order to prove that this is an isomorphism, it is hence enough to show that $h_{2 \dagger} h_{1+} \mathcal{O}_{T_{A}} \simeq h_{2 \dagger} h_{2}^{-1} k_{+} j_{\dagger} \mathcal{O}_{T_{A}} \rightarrow$ $k_{+} j_{\dagger} \mathcal{O}_{T_{A}}$ is an isomorphism. Using the triangle

$$
h_{2 \dagger} h_{2}^{-1} k_{+} j_{\dagger} \mathcal{O}_{T_{A}} \longrightarrow k_{+} j_{\dagger} \mathcal{O}_{T_{A}} \longrightarrow i_{0+} i_{0}^{\dagger} k_{+} j_{\dagger} \mathcal{O}_{T_{A}} \stackrel{+1}{\longrightarrow},
$$


it remains to show that $i_{0+} i_{0}^{\dagger} k_{+} j_{\dagger} \mathcal{O}_{T_{A}}$ is zero. For this we observe that

$$
h_{2}^{+} k_{+} j_{\dagger} \mathcal{O}_{T_{A}} \simeq h_{1+} j^{+} j_{\dagger} \mathcal{O}_{T_{B}} \simeq h_{1+} \mathcal{O}_{T_{B}}
$$

is the restriction of a quasi-equivariant module. This shows, via Lemma 3.4, that $k_{+} j_{\dagger} \mathcal{O}_{T_{A}}$ is $\mathbb{G}_{m}$-quasi-equivariant. We therefore have

$$
i_{0+} i_{0}^{\dagger} k_{+} j_{\dagger} \mathcal{O}_{T_{A}} \simeq i_{0+} \pi_{+} k_{+} j_{\dagger} \mathcal{O}_{T_{A}} \simeq i_{0+} p_{0+} p_{+} j_{\dagger} \mathcal{O}_{T_{A}} \simeq i_{0+} p_{0+} i^{\dagger} j_{\dagger} \mathcal{O}_{T_{A}},
$$

using Lemma 3.3 to substitute $p_{+}$by $i^{!}$. Since $i^{\dagger} j_{\dagger} \mathcal{O}_{T_{A}}$ is zero, the claim follows.

Consider the diagram

$$
\mathbb{A}^{1} \stackrel{F}{\longleftarrow} T_{B} \times \mathbb{A}^{n+1} \stackrel{q}{\longrightarrow} \mathbb{A}^{n+1}
$$

where $q$ is the projection and

$$
F\left(t_{1}, \ldots, t_{n}, \lambda_{0}, \ldots, \lambda_{n}\right)=\lambda_{0}+\sum_{i=1}^{n} \lambda_{i} t^{\mathbf{b}_{i}} .
$$

Denote $\Gamma=\operatorname{Var}(F)$ and write

$$
i_{\Gamma}: \Gamma \subset T_{B} \times \mathbb{A}^{n+1}, \quad j_{U}: U \rightarrow T_{B} \times \mathbb{A}^{n+1}
$$

for the inclusion of $\Gamma$ and its complement $U$. The Gauß-Manin system $q_{U+} \mathcal{O}_{U}$ is of interest since it carries a mixed Hodge structure by Saito's work in [Sai90]. Our article gives evidence to our belief that many $D$-modules arising from Euler-Koszul complexes also carry such structure, and that they relate to interesting geometric information.

Proposition 3.8. With $u=1, \delta, 1 / t, \mathfrak{H}$ and $\hat{u}=\delta, 1, \mathfrak{H}, 1 / t$, and with $k$ as in (3.3.2) we have the following isomorphisms

$$
\operatorname{FL}\left(k_{+} p^{+}\left(\mathcal{D}_{\hat{\mathbb{A}}^{1}} \bullet \hat{u}\right)\right) \simeq q_{+} F^{+}\left(\mathcal{D}_{\mathbb{A}^{1}} \bullet u\right) .
$$

Proof. Consider the diagram

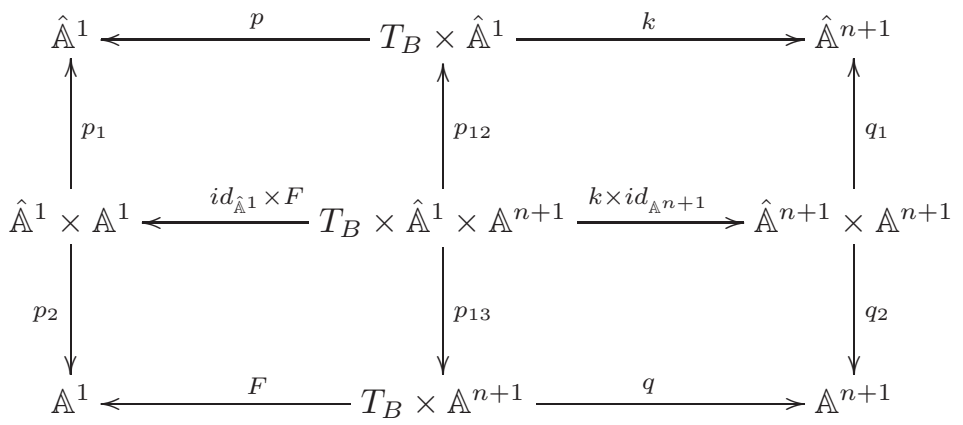

where $p_{i j}$ are the projections to the factors $i$ and $j$. Recall the Fourier-Laplace sheaf $\mathcal{L}$ on $\mathbb{A}^{n+1}$ from Definition 1.5 and denote $\mathcal{L}_{1}$ the Fourier-Laplace sheaf on 
$\mathbb{A}^{1} \times \mathbb{A}^{1}$. Then

$$
\begin{aligned}
\mathrm{FL}\left(k_{+} p^{+}\left(\mathcal{D}_{\hat{\mathbb{A}}^{1}} \bullet \hat{u}\right)\right) & =q_{2+}\left(\left(q_{1}^{+} k_{+} p^{+}\left(\mathcal{D}_{\hat{\mathbb{A}}^{1}} \bullet \hat{u}\right)\right) \otimes \mathcal{L}\right) \\
& \simeq q_{2+}\left((k \times i d)_{+} p_{12}^{+} p^{+}\left(\mathcal{D}_{\hat{\mathbb{A}}^{1}} \bullet \hat{u}\right) \otimes \mathcal{L}\right) \\
& \simeq q_{2+}(k \times i d)_{+}\left(p_{12}^{+} p^{+}\left(\mathcal{D}_{\hat{\mathbb{A}}^{1}} \bullet \hat{u}\right) \otimes(k \times i d)^{+} \mathcal{L}\right) \\
& \simeq q_{+} p_{13+}\left((i d \times F)^{+} p_{1}^{+}\left(\mathcal{D}_{\hat{\mathbb{A}}^{1}} \bullet \hat{u}\right) \otimes(k \times i d)^{+} \mathcal{L}\right) \\
& \simeq q_{+} p_{13+}\left((i d \times F)^{+} p_{1}^{+}\left(\mathcal{D}_{\hat{\mathbb{A}}^{1}} \bullet \hat{u}\right) \otimes(i d \times F)^{+} \mathcal{L}_{1}\right) \\
& \simeq q_{+} p_{13+}(i d \times F)^{+}\left(p_{1}^{+}\left(\mathcal{D}_{\hat{\mathbb{A}}^{1}} \bullet \hat{u}\right) \otimes \mathcal{L}_{1}\right) \\
& \left.\simeq q_{+} F^{+} p_{2+} p_{1}^{+}\left(\mathcal{D}_{\hat{\mathbb{A}}^{1}} \bullet \hat{u}\right) \otimes \mathcal{L}_{1}\right) \\
& \simeq q_{+} F^{+}\left(\mathcal{D}_{\mathbb{A}^{1}} \bullet u\right) .
\end{aligned}
$$

Now consider the diagram

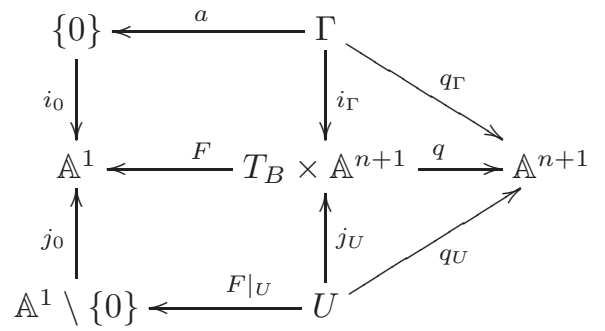

where $q$ is the projection. We have, writing $F$ for $F_{U}$,

$$
\begin{aligned}
q_{+} F^{+}\left(\mathcal{D}_{\mathbb{A}^{1}} \bullet 1 / t\right) & \simeq q_{+} F^{+} j_{0+} \mathcal{O}_{\mathbb{A}^{1} \backslash\{0\}} \simeq q_{+} j_{U+} F^{+} \mathcal{O}_{\mathbb{A}^{1} \backslash\{0\}} \simeq q_{U+} \mathcal{O}_{U}, \\
q_{+} F^{+}\left(\mathcal{D}_{\mathbb{A}^{1}} \bullet \mathfrak{H}\right) & \simeq q_{+} F^{+} j_{0 \dagger} \mathcal{O}_{\mathbb{A}^{1} \backslash\{0\}} \simeq q_{+} j_{U \dagger} F^{+} \mathcal{O}_{\mathbb{A}^{1} \backslash\{0\}} \simeq q_{+} j_{U_{\dagger}} \mathcal{O}_{U}, \\
q_{+} F^{+}\left(\mathcal{D}_{\mathbb{A}^{1}} \bullet \delta\right) & \simeq q_{+} F^{+} i_{0+} \mathcal{O}_{\{0\}} \simeq q_{+} i_{\Gamma+} a^{+} \mathcal{O}_{\{0\}} \simeq q_{+} i_{\Gamma+} \mathcal{O}_{\Gamma} \simeq q_{\Gamma+} \mathcal{O}_{\Gamma}, \\
q_{+} F^{+}\left(\mathcal{D}_{\mathbb{A}^{1}} \bullet 1\right) & \simeq q_{+} \mathcal{O}_{T_{B} \times \mathbb{A}^{n+1}},
\end{aligned}
$$

where the second isomorphism in the second line follows from the smoothness of $F$.

Notation 3.9. If $W$ is a $\mathbb{k}$-space (for example, $H_{d R}^{i}\left(T_{B} ; \mathbb{k}\right)$ ) then $\underline{W}$ denotes the trivial vector bundle $W \otimes_{\mathrm{k}} \mathcal{O}_{\mathbb{A}^{n+1}}$.

Consider the following exact sequence of $\mathcal{D}_{\hat{\mathbb{A}}^{1}}$-modules

$$
0 \longrightarrow \mathcal{D}_{\hat{\mathbb{A}}^{1}} \bullet \delta \longrightarrow \mathcal{D}_{\hat{\mathbb{A}}^{1}} \bullet \mathfrak{H} \longrightarrow \mathcal{D}_{\hat{\mathbb{A}}^{1}} \bullet 1 / t \longrightarrow \mathcal{D}_{\hat{\mathbb{A}}^{1}} \bullet \delta \longrightarrow 0
$$

Theorem 3.10. The exact sequence (3.3.3) induces an isomorphism of exact sequences

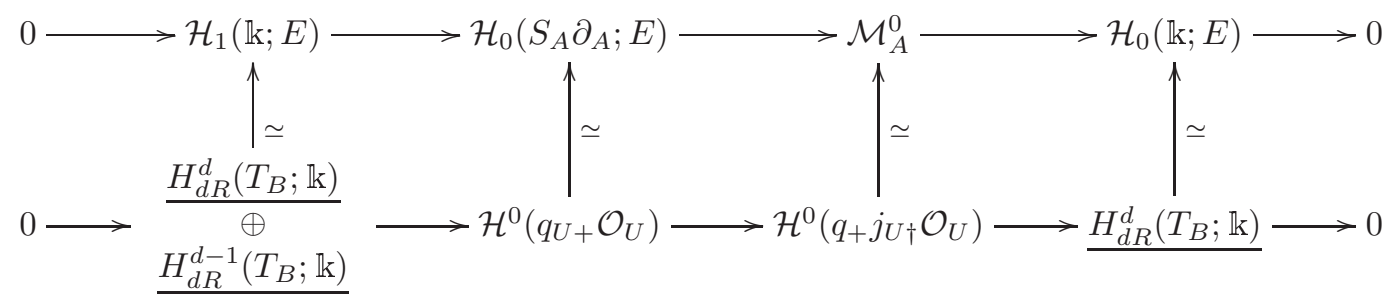


Proof. The sequence (3.3.3) is part of the long exact sequence coming from the triangle

$$
j_{0 \dagger} j_{0}^{-1} \mathcal{O}_{\hat{\mathbb{A}}^{1}} \longrightarrow j_{0+} j_{0}^{-1} \mathcal{O}_{\hat{\mathbb{A}}^{1}} \longrightarrow i_{0+} i_{0}^{\dagger} j_{0+} j_{0}^{-1} \mathcal{O}_{\hat{\mathbb{A}}^{1}} \stackrel{+1}{\longrightarrow}
$$

which is isomorphic to

$$
\mathcal{D}_{\hat{\mathbb{A}}^{1}} \bullet \mathfrak{H} \longrightarrow \mathcal{D}_{\widehat{\mathbb{A}}^{1}} \bullet 1 / t \longrightarrow\left(\mathcal{D}_{\widehat{\mathbb{A}}^{1}} \bullet \delta\right) \oplus\left(\mathcal{D}_{\widehat{\mathbb{A}}^{1}} \bullet \delta[1]\right) \stackrel{+1}{\longrightarrow}
$$

Applying the concatenated functor $\mathrm{FL} \circ k_{+} p^{+}$to the triangle above and using Lemma 3.7, Proposition 3.6, and the fact that $\mathcal{H}^{i}\left(k_{0+} \mathcal{O}_{T_{B}}\right)=\mathcal{B}_{0}\left(\begin{array}{l}d \\ i\end{array}\right)$ we obtain the upper sequence in the theorem. (Recall that $k_{0}$ sends $T_{B}$ to the origin in $\hat{\mathbb{A}}^{n+1}$ ). Applying $q_{+} F^{+} \circ \mathrm{FL}$ instead gives the lower sequence.

If one applies $q_{+} F^{+} \circ \mathrm{FL}$ to the exact sequence

$$
0 \longrightarrow \mathcal{D}_{\hat{\mathbb{A}}^{1}} \bullet 1 \longrightarrow \mathcal{D}_{\hat{\mathbb{A}}^{1}} \bullet 1 / t \longrightarrow \mathcal{D}_{\hat{\mathbb{A}}^{1}} \bullet \delta \longrightarrow 0
$$

one obtains as a part of the resulting long exact sequence the piece

$$
0 \longrightarrow \underline{H_{d R}^{d-1}\left(T_{B} ; \mathbb{k}\right)} \longrightarrow \mathcal{H}^{0}\left(q_{\Gamma+} \mathcal{O}_{\Gamma}\right) \longrightarrow \mathcal{H}^{0}\left(q_{+} j_{U \dagger} \mathcal{O}_{U}\right) \longrightarrow \underline{H_{d R}^{d}\left(T_{B} ; \mathbb{k}\right)} \longrightarrow 0,
$$

We now determine how this sequence relates to the two sequences in Theorem 3.10.

Proposition 3.11. The exact sequence (3.3.5) is the quotient of the exact sequence

$0 \longrightarrow \underline{H_{d R}^{d}\left(T_{B} ; \mathbb{k}\right)} \oplus \underline{H_{d R}^{d-1}\left(T_{B} ; \mathbb{k}\right)} \longrightarrow \mathcal{H}^{0}\left(q_{U+} \mathcal{O}_{U}\right) \longrightarrow \mathcal{H}^{0}\left(q_{+} j_{U \dagger} \mathcal{O}_{U}\right) \longrightarrow \underline{H_{d R}^{d}\left(T_{B} ; \mathbb{k}\right)} \longrightarrow 0$

by the exact sequence

$$
0 \longrightarrow \underline{H_{d R}^{d}\left(T_{B} ; \mathbb{k}\right)} \longrightarrow \underline{H_{d R}^{d}\left(T_{B} ; \mathbb{k}\right)} \longrightarrow 0 \longrightarrow 0 \longrightarrow 0 .
$$

Proof. Consider the Fourier-Laplace transforms of the sequences (3.3.3) and (3.3.5). We get a commutative diagram with exact rows and columns:

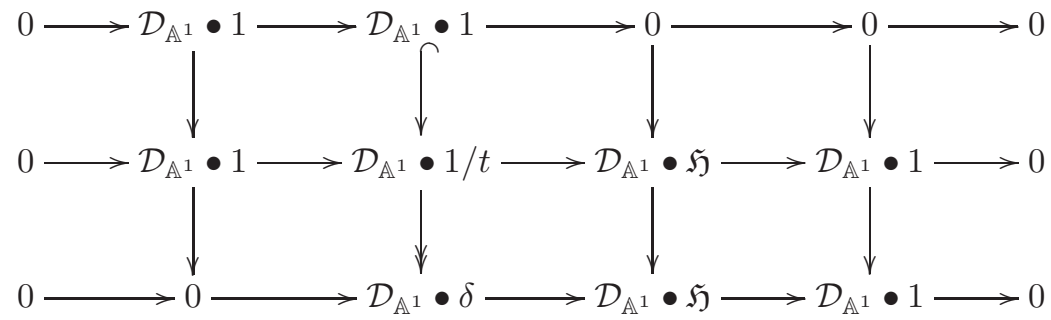

and morphisms of triangles

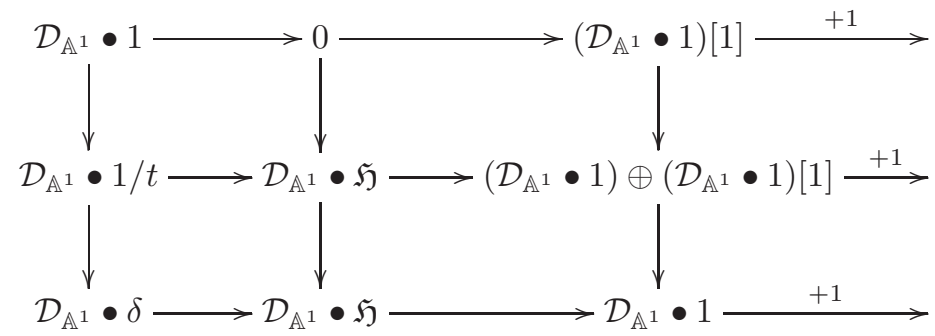


From this, we get an exact sequence of exact rows

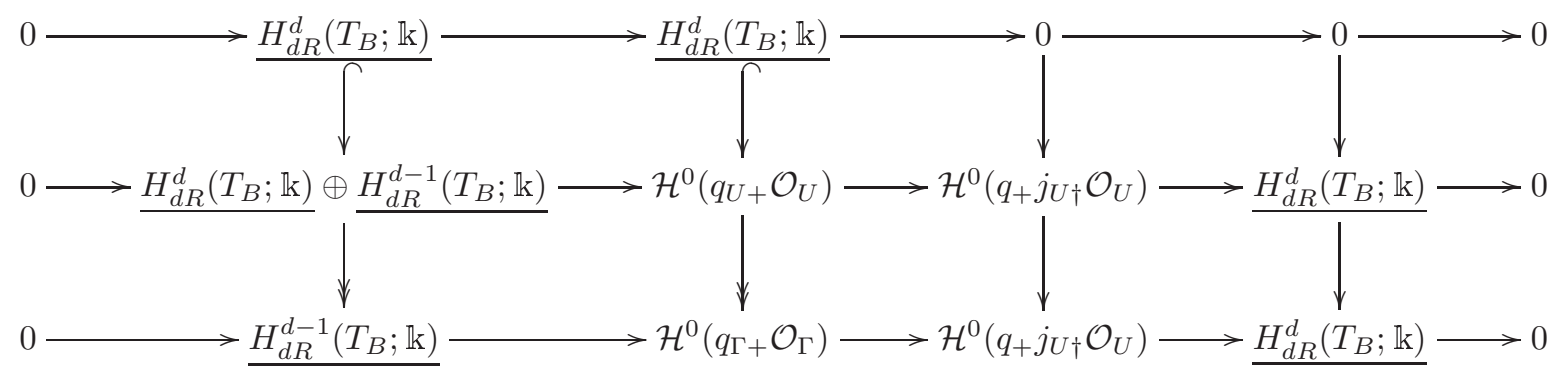

The lower middle maps are surjective since $F^{+}$is exact and $q_{+}$is right exact.

We now introduce a family of Laurent polynomials defined on $T_{B} \times \mathbb{A}^{n}$ using the columns of the matrix $B$. For this, recall Definition 3.3.1 and consider the ring homomorphism

$$
\begin{aligned}
\mathbb{k}\left[\lambda_{0}, \ldots, \lambda_{n}\right] & \longrightarrow \mathbb{k}\left[t_{1}^{ \pm}, \ldots, t_{d}^{ \pm}\right] \otimes_{\mathbb{k}} \mathbb{k}\left[\lambda_{1}, \ldots, \lambda_{n}\right] \\
\lambda_{i} & \mapsto \begin{cases}-\sum_{i=1}^{n} t^{\mathbf{b}_{i}} \otimes \lambda_{i} & \text { for } i=0 ; \\
\lambda_{i} & \text { for } i=1, \ldots, n,\end{cases}
\end{aligned}
$$

which induces a family of Laurent polynomials

$$
\varphi_{B}: T_{B} \times \mathbb{A}^{n} \longrightarrow \mathbb{A}^{n+1}=\mathbb{A}^{1} \times \mathbb{A}^{n}
$$

and an isomorphism

$$
i_{\varphi}: T_{B} \times \mathbb{A}^{n} \longrightarrow \Gamma \subseteq T_{B} \times \mathbb{A} \times \mathbb{A}^{n}
$$

onto the graph $\Gamma$. Hence $\varphi_{B}=q_{\Gamma} \circ i_{\varphi}$ and therefore $\mathcal{H}^{0}\left(\varphi_{B+} \mathcal{O}_{T_{B} \times \mathbb{A}^{n}}\right) \simeq \mathcal{H}^{0}\left(q_{\Gamma+} \mathcal{O}_{\Gamma}\right)$.

This recovers a special case of a theorem of [Rei14], i.e. there is an exact sequence

$$
0 \longrightarrow \underline{H_{d R}^{d-1}\left(T_{B} ; \mathbb{k}\right)} \longrightarrow \mathcal{H}^{0}\left(\varphi_{B+} \mathcal{O}_{T_{B} \times \mathbb{A}^{n}}\right) \longrightarrow \mathcal{M}_{A}^{0} \longrightarrow \underline{H_{d R}^{d}\left(T_{B} ; \mathbb{k}\right)} \longrightarrow 0
$$

which is isomorphic to the sequence (3.3.5).

3.4. Vanishing Gauß-Manin system and the extension class. In this section we show that the $A$-hypergeometric system is an extension of a trivial vector bundle of rank one by the quotient of a Gauß-Manin system modulo its flat sections. We show that this extension does not split.

As before, $\beta=0$ (and $A$ is saturated, homogeneous, and pointed).

Definition 3.12. The vanishing Gauß-Manin system $\mathcal{V}$ with respect to the map $\varphi_{B}$ is the cokernel of the map $\underline{H_{d R}^{d-1}\left(T_{B} \mathbb{k}\right)} \longrightarrow \mathcal{H}^{0}\left(\varphi_{B+} \mathcal{O}_{T_{B} \times \mathbb{A}^{n}}\right)$. In other words,

$$
0 \longrightarrow \mathcal{V} \longrightarrow \mathcal{M}_{A}^{0} \longrightarrow \underline{H_{d R}^{d}\left(T_{B} ; \mathbb{k}\right)} \longrightarrow 0
$$

is exact. We write $V_{A}=\Gamma\left(\mathbb{A}^{n+1}, \mathcal{V}_{A}\right)$ and note the short exact sequence

$$
0 \longrightarrow V_{A} \longrightarrow M_{A}^{0} \longrightarrow O_{A} \longrightarrow 0 .
$$


The terminology is borrowed from the vanishing cohomology of a hyperplane section $j: X \hookrightarrow Y$ of an $n$-dimensional projective variety $Y$ which is a direct summand $H^{n-1}(X)=H^{n-1}(X)_{v a n} \oplus j^{*} H^{n-1}(Y)$.

The sheaf $\mathcal{V}$ appears perhaps for the first time in Stienstra's article [Sti98, Formula (61)], essentially as a restriction of (3.4.1) to the smooth locus (where all sheaves in (3.3.5) become vector bundles). However, our situation is more general even in Stienstra's set-up since in [Sti98] the matrix B is assumed to be homogeneous while it is arbitrary for us.

A natural question is: what is the extension class of $\mathcal{M}_{A}^{0}$ inside the sequence (3.4.1)? Our next result answers this question, confirming a prediction of Duco van Straten.

Theorem 3.13. Write $\mathcal{O}$ for $H_{d R}^{d}\left(T_{B} ; \mathbb{k}\right) \otimes \mathcal{O}_{\mathbb{A}^{n+1}}$. There are natural (in $\mathbb{k}$ ) isomorphisms

$$
\operatorname{Ext}_{\mathcal{D}}^{i}(\mathcal{O}, \mathcal{V}) \simeq \begin{cases}\mathbb{k} & \text { for } i=1 \\ 0 & \text { else. }\end{cases}
$$

The class of the sequence (3.4.1) is nonzero and induced by the identity on $\mathcal{O}$ under the connecting morphism.

Proof. Since $\mathbb{A}^{n+1}$ is affine it suffices to compute on the level of global sections. By Corollary 2.12, $\operatorname{Ext}_{D_{A}}\left(O_{A}, M_{A}^{\beta}\right)$ vanishes for $\beta \in \mathbb{N} A$. Hence, $\operatorname{Ext}_{D_{A}}^{i}\left(O_{A}, V_{A}\right)=$ $\operatorname{Ext}_{D_{A}}^{i-1}\left(O_{A}, O_{A}\right)$ and so has exactly the prescribed $\mathbb{k}$-space structure. In particular, (3.4.1) does not split.

The class of (3.4.1) inside $\operatorname{Ext}_{D}^{1}\left(O_{A}, V\right) \simeq \operatorname{Ext}_{D}^{0}\left(O_{A}, O_{A}\right)$ is the image of the identity on $\mathcal{O}_{A}$ under the connecting morphism induced by (3.4.1), compare [Wei94, Sec. 3.4]. Since the connecting morphism is an isomorphism, this element is nontrivial.

\section{REFERENCES}

[Ber] Joseph Bernstein, Algebraic theory of D-modules., Unpublished notes available online at http://www.math.uchicago.edu/arinkin/langlands/Bernstein/Bernstein-dmod.pdf. 2.2

$\left[\mathrm{BGK}^{+} 87\right]$ A. Borel, P.-P. Grivel, B. Kaup, A. Haefliger, B. Malgrange, and F. Ehlers, Algebraic D-modules, Perspectives in Mathematics, vol. 2, Academic Press Inc., Boston, MA, 1987. 2.2

[BH93] Winfried Bruns and Jürgen Herzog, Cohen-Macaulay rings, Cambridge Studies in Advanced Mathematics, vol. 39, Cambridge University Press, Cambridge, 1993. MR 1251956 (95h:13020) 2.4

[Bry86] Jean-Luc Brylinski, Transformations canoniques, dualité projective, théorie de Lefschetz, transformations de Fourier et sommes trigonométriques, Astérisque (1986), no. 140-141, 3-134, 251, Géométrie et analyse microlocales. 1

[DE03] Andrea D'Agnolo and Michael Eastwood, Radon and Fourier transforms for $\mathcal{D}$ modules, Adv. Math. 180 (2003), no. 2, 452-485. 3.3

[GGZ87] I. M. Gel/fand, M. I. Graev, and A. V. Zelevinskiŭ, Holonomic systems of equations and series of hypergeometric type, Dokl. Akad. Nauk SSSR 295 (1987), no. 1, 14-19. MR 9029361

[Gro66] A. Grothendieck, On the de Rham cohomology of algebraic varieties, Inst. Hautes Études Sci. Publ. Math. (1966), no. 29, 95-103. MR 01991942.3

[GZK89] I. M. Gel/fand, A. V. Zelevinskǐ̌, and M. M. Kapranov, Hypergeometric functions and toric varieties, Funktsional. Anal. i Prilozhen. 23 (1989), no. 2, 12-26. MR 10113531

[HTT08] Ryoshi Hotta, Kiyoshi Takeuchi, and Toshiyuki Tanisaki, D-modules, perverse sheaves, and representation theory, Progress in Mathematics, vol. 236, Birkhäuser Boston, 
Inc., Boston, MA, 2008, Translated from the 1995 Japanese edition by Takeuchi. MR $23573611.2, \bullet(a)$

$\left[\mathrm{ILL}^{+} 07\right]$ Srikanth B. Iyengar, Graham J. Leuschke, Anton Leykin, Claudia Miller, Ezra Miller, Anurag K. Singh, and Uli Walther, Twenty-four hours of local cohomology, Graduate Studies in Mathematics, vol. 87, American Mathematical Society, Providence, RI, 2007. MR 2355715 (2009a:13025) 2.1

[KS97] Masaki Kashiwara and Pierre Schapira, Integral transforms with exponential kernels and Laplace transform, J. Amer. Math. Soc. 10 (1997), no. 4, 939-972. MR 1447834 1.5

[MMW05] Laura Felicia Matusevich, Ezra Miller, and Uli Walther, Homological methods for hypergeometric families, J. Amer. Math. Soc. 18 (2005), no. 4, 919-941 (electronic). MR 2163866 (2007d:13027) 1, 1.1, 2.2, 2.2, 2.4, 2.4, •(c), 3

[Rei14] Thomas Reichelt, Laurent Polynomials, GKZ-hypergeometric Systems and Mixed Hodge Modules, Compositio Mathematica (150) (2014), 911-941. 1, 3.3

[RSW17] Thomas Reichelt, Christian Sevenheck, and Uli Walther, On the b-functions of hypergeometric systems, Int. Math. Res. Not. (to appear 2017), 1-14. 3.1

[Sai90] Morihiko Saito, Mixed Hodge modules, Publ. Res. Inst. Math. Sci. 26 (1990), no. 2, 221-333. MR 10474153.3

[SST00] Mutsumi Saito, Bernd Sturmfels, and Nobuki Takayama, Gröbner deformations of hypergeometric differential equations, Algorithms and Computation in Mathematics, vol. 6, Springer-Verlag, Berlin, 2000. MR 1734566 (2001i:13036) 2.5

[ST98] Bernd Sturmfels and Nobuki Takayama, Gröbner bases and hypergeometric functions, Gröbner bases and applications (Linz, 1998), London Math. Soc. Lecture Note Ser., vol. 251, Cambridge Univ. Press, Cambridge, 1998, pp. 246-258. MR 1708882 (2001c:33026) 2.7

[Sti98] Jan Stienstra, Resonant hypergeometric systems and mirror symmetry, Integrable systems and algebraic geometry (Kobe/Kyoto, 1997), World Sci. Publ., River Edge, NJ, 1998, pp. 412-452. MR 1672077 3, 3.4

[SW08] Mathias Schulze and Uli Walther, Irregularity of hypergeometric systems via slopes along coordinate subspaces, Duke Math. J. 142 (2008), no. 3, 465-509. MR 2412045 (2009b:13067) 1.4

[SW09] - Hypergeometric D-modules and twisted Gauß-Manin systems, J. Algebra 322 (2009), no. 9, 3392-3409. MR 2567427 (2010m:14028) 1, 1.1, 1, 2.2, 2.2, 2.3, 2.3

[Wei94] Charles A. Weibel, An introduction to homological algebra, Cambridge Studies in Advanced Mathematics, vol. 38, Cambridge University Press, Cambridge, 1994. MR 1269324 (95f:18001) 3.4

T. Reichelt, Mathematisches Institut, Universität Heidelberg, Im Neuenheimer Feld 205, 69120 Heidelberg, Germany

E-mail address: treichelt@mathi.uni-heidelberg.de

U. Walther, Purdue University, Dept. of Mathematics, 150 N. University St., West LAFAYETTE, IN 47907, USA

E-mail address: walther@math.purdue.edu 